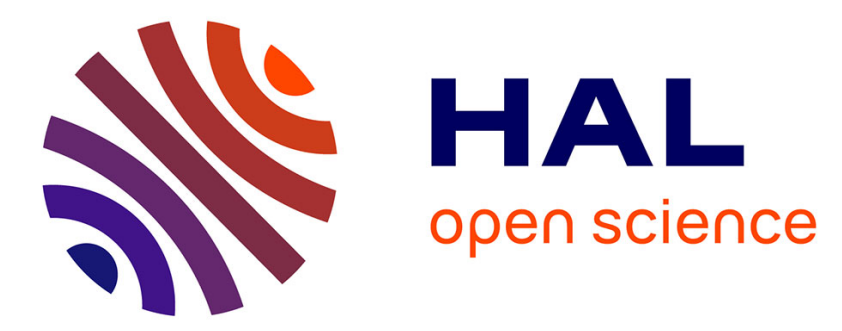

\title{
Impact of the Formulation Pathway on the Colloidal State and Crystallinity of Poly- $\epsilon$-caprolactone Particles Prepared by Solvent Displacement
}

Carlotta Pucci, Fabrice Cousin, François Dole, Jean-Paul Chapel, Christophe Schatz

\section{To cite this version:}

Carlotta Pucci, Fabrice Cousin, François Dole, Jean-Paul Chapel, Christophe Schatz. Impact of the Formulation Pathway on the Colloidal State and Crystallinity of Poly- $\epsilon$ caprolactone Particles Prepared by Solvent Displacement. Langmuir, 2018, 34 (7), pp.2531-2542. 10.1021/acs.langmuir.7b04198 . hal-01756032

\section{HAL Id: hal-01756032 \\ https://hal.science/hal-01756032}

Submitted on 5 Apr 2018

HAL is a multi-disciplinary open access archive for the deposit and dissemination of scientific research documents, whether they are published or not. The documents may come from teaching and research institutions in France or abroad, or from public or private research centers.
L'archive ouverte pluridisciplinaire HAL, est destinée au dépôt et à la diffusion de documents scientifiques de niveau recherche, publiés ou non, émanant des établissements d'enseignement et de recherche français ou étrangers, des laboratoires publics ou privés. 


\section{Impact of the formulation pathway on the colloidal state and crystallinity of poly-e-caprolactone particles prepared by solvent displacement}

Carlotta Pucci, ${ }^{a, b^{*}}$ Fabrice Cousin, ${ }^{c}$ François Dole, ${ }^{a}$ Jean-Paul Chapel, ${ }^{a *}$ and Christophe Schatz $b^{b^{*}}$

${ }^{a}$ Centre de Recherche Paul Pascal (CRPP), UPR CNRS 8641, Université Bordeaux, 33600 Pessac, France

${ }^{\mathrm{b}}$ Laboratoire de Chimie des Polymères Organiques (LCPO), Université de Bordeaux, CNRS, Bordeaux INP, UMR 5629, 33600 Pessac, France

${ }^{\mathrm{c}}$ Laboratoire Léon Brillouin, CEA Saclay, 91191 Gif sur Yvette Cedex, France. 


\section{ABSTRACT}

The formulation pathway and/or mixing method are known to be relevant in many out-of equilibrium processes. In this work we studied the effect of the mixing conditions on the physicochemical properties of poly-E-caprolactone (PCL) particles prepared by solvent displacement. More specifically, water was added in one shot (fast addition) or drop-by-drop to PCL solution in THF to study the impact of the mixing process on particle properties including size, stability and crystallinity. Two distinct composition maps representing the Ouzo domain characteristic of the presence of metastable nanoparticles have been established for each mixing method. Polymer nanoparticles are formed in the Ouzo domain according to a nucleation and growth (or aggregation) mechanism. The fast addition promotes a larger nucleation rate thus favoring the formation of small and uniform particles. For the drop-by-drop addition, for which the polymer solubility gradually decreases, the composition trajectories systematically cross an intermediate unstable region between the solubility limit of the polymer and the Ouzo domain. This leads to heterogeneous nucleation as shown by the formation of larger and less stable particles. Particles formed in the Ouzo domain have semi-crystalline properties. The PCL melting point is decreased with the THF fraction trapped in particles in accordance with Flory's theory for melt crystallization. On the other hand, the degree of crystallinity is constant, around $20 \%$ regardless the THF fraction. No difference between fast and slow addition could be detected on the semi-crystalline properties of the particles which emphasize that thermodynamic rather than kinetic factors drive the polymer crystallization in particles. The recovery of bulk PCL crystallinity after the removal of THF from particles tends to confirm this hypothesis. 


\section{INTRODUCTION}

Polymer nanoparticles are appealing materials for several applications, such as therapeutics, bioengineering, imaging, optics and drug delivery. ${ }^{1-4}$ In the last decades great attention has been paid to their rational design through various non-covalent approaches, in order to target the synthesis to a specific product. Polymer self-assembly is one of the most common strategies at the moment. However, most of the processes involving non-covalent polymer assembly occur under non-equilibrium conditions and the predictive power on the final assembly is low. The mechanism of out-of-equilibrium polymer self-assembly is still not fully clarified, especially concerning the thermodynamics and the kinetics aspects. ${ }^{5}$ When the aggregation is kinetically driven, the local environment (polymer concentration, the mixing rate and order of addition, for example) has a great impact on the interactions between the components, modifying the morphology, the properties and the stability of the resulting assemblies. ${ }^{6-11}$ Such dependence is known ${ }^{12}$ but has never been investigated in a comprehensive manner. However, this is a fundamental aspect in nanotechnology, where a rational design of the product is required for future industrial developments.

Among current approaches to design polymer nanoparticles, the nanoprecipitation or solvent displacement method has become a standard method, especially to entrap or encapsulate active principles. $^{13-16}$ The procedure consists in dissolving the polymer and the drug into a watermiscible solvent, like THF or acetone, and then mixing the polymer solution with an excess of water to trigger the spontaneous formation of polymer nanoparticles. The supersaturation defined as the ratio between the actual polymer concentration and the polymer solubility in the solvent mixture is the driving force of the nanoprecipitation, since it determines the nucleation rate. Once the first nuclei are formed, they can grow either through association with solute molecules in the 
bulk (nucleation-and-growth mechanism) or through particle aggregation (nucleation-andaggregation mechanism), the latter being favored in high supersaturation conditions. ${ }^{16}$ An alternate process referred as 'emulsification-diffusion' based on the breaking-up of polymer solution into submicron droplets followed by the polymer nanoprecipitation when the solvent flows away from droplets has also been proposed, especially when the precipitation occurs at the highest polymer concentration and organic/aqueous phase ratio. ${ }^{17}$ Particle stabilization can be achieved by the charges at particle surface when the polymer contains ionizable groups, or through the addition of a surfactant in the medium, both limiting further growth and/or aggregation of particles. ${ }^{14}$

Nanoprecipitation is a non-equilibrium process; variations of initial mixing conditions may therefore lead to different results. In a typical situation where an excess of water is added to a polymer solution, the generation of supersaturation can be low and nucleation rates may be slow relative to growth under poor mixing conditions, leading to the formation of large polydisperse particles. ${ }^{15}$ Conversely, good mixing conditions create local high supersaturation and high nucleation rates resulting in the formation of smaller particles. A large body of works has already been devoted on how the mixing conditions can be tuned to obtain particles small in size and with a narrow size distribution. ${ }^{14,15,18}$ In addition, other parameters like the solvent/water miscibility, the solvent/water ratio, the polymer concentration, the polymer molecular weight and the addition of surfactants have also been considered. 16-20

In this work we aim at studying another aspect related to polymer nanoprecipitation, namely the nanoparticle crystallinity, which is a fundamental parameter in many applications, especially in drug delivery, since it affects directly the drug release efficiency. ${ }^{21}$ In addition, it is a useful tool to monitor the aggregation process, as it is related to the way the polymer chains interact 
each other and with the solvent molecules. A few works have addressed this question but no systematic studies have been carried out on the impact of the conditions of nanoprecipitation and related particle sizes on the particle crystallinity. It has been reported that the nanoprecipitation lowers the crystallinity of the polymer ${ }^{22}$ but no effect of the particle size in the $200-400 \mathrm{~nm}$ range has been clearly evidenced on the melting temperature of the polymer. ${ }^{22}$ A strong decrease of the particle crystallinity has been however reported for smaller polymer particles $(<100 \mathrm{~nm})$ prepared through nanoprecipitation, but this was attributed to the use of surfactants to obtain smaller nanoparticles. ${ }^{23}$ Here, we specifically aim at investigating the effect of mixing conditions on the final crystallinity degree by applying two ways of mixing as originally proposed by Aubry et al: $:^{16}$ the fast (FA) and the drop-by-drop (DD) addition of water into the polymer solution in order to vary the mixing time and consequently the conditions of supersaturation.

The well-studied poly(e-caprolactone) (PCL), which is a hydrophobic and semi-crystalline polymer, was used as a model system. PCL is widely employed in biomedical applications owing to its well-established biocompatibility, nontoxicity and biodegradability. ${ }^{24-26}$ With a low melting point around $60^{\circ} \mathrm{C}$, it is possible to perform direct thermal analyses of PCL in aqueous solutions through micro differential scanning calorimetry ( $\mu \mathrm{DSC})$ measurements rather than on dried nanoparticles. It has been shown that the semi-crystalline structure of PCL is preserved to some extent in nanoparticles obtained through the solvent displacement method. ${ }^{27}$

It is important to note that the primary aim of this work is to better understand the polymer nanoprecipitation from a general point of view. The choice of the two mixing methods is then not driven by the need of obtaining an optimized formulation of PCL nanoparticles for a particular application. The fast addition of water into the organic polymer solution ensures a fast change in solvent quality for the polymer whereas, the drop-by-drop addition (in the same order) 
enables to tune the solvent quality very slowly, allowing the polymer to rearrange in a state closer to equilibrium. We can then compare two opposite situations and directly point out the impact of the initial mixing conditions on the final crystallinity and other key parameters of the PCL NPs. For the same fundamental reason, all nanoprecipitation experiments were carried out from PCL solutions without any surfactant, so that neither the formation of particles nor the polymer crystallization mechanism could be affected by the presence of additives. ${ }^{23,27,28}$

\section{MATERIALS AND METHODS}

\section{CHEMICALS}

The poly- $\varepsilon$-caprolactone PCL of $\mathrm{M}_{\mathrm{w}} 45 \mathrm{kDa}$ and $2 \mathrm{kDa}$ were purchased from Sigma Aldrich and used as received. PCL chains certainly have at least one $\mathrm{OH}$ function at their ends. THF (Sigma Aldrich), $\mathrm{d}_{8}$-THF and $\mathrm{D}_{2} \mathrm{O}$ (Euriso-top) were of analytical grade and used as received. Water was pretreated with a Millipore Elix 10 system.

\section{COMPOSITION MAP (CM) DETERMINATION}

The composition map of the PCL/THF/ $\mathrm{H}_{2} \mathrm{O}$ system prepared by solvent displacement was determined to find the composition range where nanoparticles are formed. Water was added to the PCL/THF organic solution following two procedures: i) Fast addition (FA): high-speed manual injection with a syringe; ii) Drop-by-drop (DD): injection controlled by a syringe pump (Harvard Apparatus PHD 4400 Programmable) at $0.3 \mathrm{ml} / \mathrm{min}$. The stirring speed was set at 200 rpm and stopped right after mixing. We emphasize that both of these methods have been extensively reported in the context of nanoprecipitation. ${ }^{16,29}$ Each area in the CM was identified 
at first by visual inspection. A bluish appearance of the samples was related to the presence of nanoparticles $\sim 200 \mathrm{~nm}$ (Tyndall effect). A milky aspect, instead, was ascribed to either a high concentration of nanoparticles or to the presence of larger microparticles. ${ }^{30}$ In order to discriminate between these two possibilities samples were further analyzed by centrifugation (3900 x g for $30 \mathrm{~min})$. According to Aubry et al., ${ }^{16}$ centrifuging a sample with microparticles should lead to sediment that cannot be redisperse by vortex. Conversely, when there are only nanoparticles, the sedimented powder can be easily redispersed. This is because microparticles

coalesce more easily than nanoparticles under the pressure of the centrifugation. ${ }^{16}$ The binodal line, defined as the equilibrium phase separation boundary, was determined by titrating a solution of PCL in THF with water until the transparent sample turned turbid. ${ }^{16}$ Finally the actual size of the particles was confirmed by DLS and SEM measurements (see below).

\section{PREPARATION OF PCL PARTICLES}

After determining the composition map, four samples having the same final PCL content $(0.18 \% \mathrm{w} / \mathrm{w})$ but different final THF volume fractions $\left(\mathrm{X}_{\mathrm{THF}}=0.1,0.2,0.3\right.$ and 0.4$)$ were selected for further characterizations. PCL particles were prepared from stock solutions of the polymer dissolved in THF at different concentrations $(2.0,1.0,0.7$ and $0.5 \% \mathrm{w} / \mathrm{w})$. Each formulation was prepared according to the FA and DD approaches. As in some cases incipient sedimentation was observed, the final PCL dispersion concentration was systematically determined by thermogravimetric analysis (TGA) prior to perform $\mu \mathrm{DSC}$ experiments. When needed, THF from the THF/water solution was removed by dialysis against water using a FloatA-lyzer (Spectrum Labs, MWCO $20 \mathrm{kDa}$ ). 


\section{THERMOGRAVIMETRIC ANALYSIS}

TGA was performed using a Q50 Thermogravimetric Analyzers (TA instruments) to determine the final concentration of PCL dispersions. About $45 \mathrm{mg}$ of sample was inserted in an aluminum pan and then heated from room temperature to $200^{\circ} \mathrm{C}$ at a heating rate of $5^{\circ} \mathrm{C} / \mathrm{min}$. The final temperature was kept isothermal for 10 minutes to allow the solvent to completely evaporate.

\section{SEM}

The particle size and morphology was investigated using scanning electron microscopy (SEM). Samples were prepared by casting a drop of the dispersion on a coverslip and letting it evaporate at room temperature. The resulting film was then coated with thin layer of Gold/Palladium with a JEOL JFC-1200 coater and finally analyzed with a field emission scanning electron microscope (JEOL JSM 6700F) with a voltage of $5 \mathrm{kV}$.

\section{DYNAMIC AND STATIC LIGHT SCATTERING}

Dynamic (DLS) and static (SLS) light scattering experiments were performed using an ALV laser goniometer, with a $22 \mathrm{~mW}$ linearly polarized laser $(632.8 \mathrm{~nm} \mathrm{HeNe})$ and an ALV5000/EPP multiple tau digital correlator with a 125 ns initial sampling time. The DLS autocorrelation functions were obtained at a scattering angle of $90^{\circ}$ and a temperature of $25^{\circ} \mathrm{C}$. The intensity-weighted relaxation time distributions were obtained using the multi-exponential CONTIN processing method. A single relaxation mode corresponding to the translational diffusion of particles was usually found on each distribution. The hydrodynamic radius $\left(R_{H}\right)$ of the particles was calculated using the Stokes-Einstein relation:

$$
R_{H}=\frac{k_{B} T}{6 \pi \eta D_{a p p}}
$$


where $k_{B}$ is the Boltzmann constant, $T$ the temperature, $\eta$ the viscosity of the solvent mixture and $D_{\text {app }}$ the apparent diffusion coefficient of particles determined from the main relaxation time ( $\tau$ ) on size distribution, $\mathrm{D}_{\text {app }}=\left(1 / \tau \mathrm{q}^{2}\right)$ where $q$ is the scattering vector (see below). The viscosities and refractive indices of the different $\mathrm{THF} / \mathrm{H}_{2} \mathrm{O}$ mixtures were obtained by fitting experimental data reported in literature. ${ }^{31}$

SLS experiments were carried out at $25^{\circ} \mathrm{C}$ by varying the scattering angle from $25^{\circ}$ to $150^{\circ}$ with a $5^{\circ}$ stepwise increase. The solvent scattering intensities were acquired in same conditions and subtracted from the sample scattering intensities; the result was then normalized from the scattering intensity of the toluene and multiplied by the Rayleigh ratio of toluene $\left(1.366 .10^{-5} \mathrm{~cm}^{-1}\right.$ at $632.8 \mathrm{~nm})$ and $\left(\mathrm{n} / \mathrm{n}_{\text {toluene }}\right)^{2}$ (Herman \& Levinson correction of the scattering volume for cylindrical cuvette) to obtain the Rayleigh ratio as a function of the scattering vector defined as:

$$
q=\frac{4 \pi n}{\lambda_{0}} \sin \left(\frac{\theta}{2}\right)
$$

where $\lambda_{0}$ is the wavelength of the incident beam in vacuum, $n$ is the refractive index of the solvent, and $\theta$ is the scattering angle. The data were merged with the small angle neutron scattering results to get the scattering curves over the whole range of $q$.

\section{ZETA POTENTIAL}

Particle zeta potential was evaluated by a Zetasizer Nano ZS (Malvern Instr., UK). Measurements were carried out using -a universal "dip" cell for samples in $\mathrm{THF} / \mathrm{H}_{2} \mathrm{O}$ mixtures. $\mathrm{NaCl} 1 \mathrm{mM}$ was added to particle dispersions prior to run zeta analysis. The dielectric constant and viscosity values for the different solvent mixtures were taken into account to determine the zeta potential from the electrophoretic mobility. 


\section{DIFFERENTIAL SCANNING MICROCALORIMETRY}

Differential scanning microcalorimetry ( $\mu \mathrm{DSC}$ ) experiments on nanoparticle dispersions were performed using a Setaram Micro DSC III VP-DSC microcalorimeter. The samples were scanned from 10 to $50{ }^{\circ} \mathrm{C}$ at a heating rate of $1{ }^{\circ} \mathrm{C}$ per minute. A second scan was performed in the same conditions $\left(10-50^{\circ} \mathrm{C}, 1^{\circ} \mathrm{C} / \mathrm{min}\right)$ in some cases. The typical volume of the particle dispersion used for each experiment was $500 \mu \mathrm{L}$. The cells were closed tightly so that water and THF could not evaporate during the experiment. A mixture of THF and water was used as reference so that the $\mathrm{THF} / \mathrm{H}_{2} \mathrm{O}$ volume ratio matched the solvent composition in the corresponding samples. All the thermograms were normalized by the polymer weight in the sample (using the mass concentrations obtained by TGA) and reported in W/g. The enthalpy of fusion $\left(\Delta \mathrm{H}_{\mathrm{f}}\right)$ for the melting process was determined by integrating the area of the melting peak. The mass degree of crystallinity, $\mathrm{X}_{\mathrm{c}}$, of each sample was calculated by dividing the experimental $\Delta \mathrm{H}_{\mathrm{f}}(\mathrm{J} / \mathrm{g})$ by the heat of fusion of pure crystalline polycaprolactone $(139.5 \mathrm{~J} / \mathrm{g}) .^{32,33}$ As a reference, the bulk polymers were also analyzed in the same conditions (see Figure S1).

\section{NUCLEAR MAGNETIC RESONANCE SPECTROSCOPY}

Proton nuclear magnetic resonance spectroscopy $\left({ }^{1} \mathrm{H}-\mathrm{NMR}\right)$ (Bruker AC-400 spectrometer) was used to quantify the THF content trapped inside PCL particles during the nanoprecipitation process. The nanoparticle dispersions were first centrifuged at 1500 x g for 10 minutes. The supernatant was removed and the particles quickly washed with water to remove any traces of THF. Then, the particles were dissolved in deuterated chloroform $\left(\mathrm{CDCl}_{3}\right)$ in which both PCL and THF are miscible. Two reference peaks, one for PCL and the other for THF, were selected to determine integral ratios from which PCL to THF molar and mass ratios can be derived (see 
Figure S2 for more details). From known THF and PCL densities at $25^{\circ} \mathrm{C}$, the volume ratios could be derived.

\section{SMALL ANGLE NEUTRON SCATTERING}

Small angle neutron scattering (SANS) measurements were performed on the PAXY spectrometer at the Laboratoire Léon Brillouin (Saclay, France). The experiments were done by using the contrast variation method, that is, by varying the deuteration percentage in the solvent mixtures. While keeping a constant THF-to-water volume ratio of 0.20 , the polymer particles from PCL 45kDa were prepared according to previously described FA and DD approaches in the following solvent mixtures: 1) THF-D/ $\mathrm{H}_{2} \mathrm{O}=20 / 80 ;$ 2) THF/ $\mathrm{D}_{2} \mathrm{O}=20 / 80 ; 3$ ) THF-D/ $\mathrm{D}_{2} \mathrm{O} / \mathrm{H}_{2} \mathrm{O}$ $\left.=20 / 65 / 15 ; 4) \mathrm{THF} / \mathrm{H}_{2} \mathrm{O}=20 / 80 ; 5\right) \mathrm{THF} / \mathrm{D}_{2} \mathrm{O} / \mathrm{H}_{2} \mathrm{O}=20 / 65 / 15$. The mixtures 1 and 5 matched the scattering length density of the PCL and the THF in particles, respectively. For centrosymmetrical objects likes spheres, the scattering intensity is described by the relation:

$$
I=\phi_{P}\left(\rho_{P}-\rho_{\text {solvent }}\right)^{2} V_{p} P(q) S(q)
$$

where $\phi_{P}$ is the volume fraction of the particles, $\rho_{P}$ and $\rho_{\text {solvent }}$ are the scattering length densities of the particles and the solvent, $V_{p}$ the particle volume, $P(q)$ is the form factor and $S(q)$ the structure factor which can be approximated to $S(q)=1$ since the particle dispersion is very diluted. $\rho_{P}$ can be written as:

$$
\rho_{P}=\phi_{P C L} \rho_{P C L}+\phi_{T H F, \text { inside }} \rho_{T H F}=\left(1-\phi_{T H F, \text { inside }}\right) \rho_{P C L}+\phi_{T H F, \text { inside }} \rho_{T H F}
$$

where $\rho_{P C L}$ and $\rho_{T H F}$ are the scattering length densities of the PCL and THF, whereas $\phi_{P C L}$ and $\phi_{T H F \text {,inside }}$ are the volume fraction of PCL and THF trapped inside the particles. Therefore, the particle scattering density depends on the THF trapped inside. For the same sample, $I$ depends only on $\rho_{\text {solvent }}$ and consequently can be modified by changing the solvent contrast. The volume 
fraction of THF inside the particles was then determined by varying this value until the theoretical normalized intensities of the solvent mixture series matched the experimental ones. The scattering curves for the samples prepared by DD in the solvent mixtures 1 and 4 were discarded. Indeed, the particles prepared under such condition have a poor stability; therefore the time devoted to measurements for a given sample was limited by the sedimentation rate. For these two contrast conditions, the scattered intensity was too low in the measurement window to allow a proper measurement with a good signal-to-noise ratio.

\section{X-RAY SCATTERING}

X-Ray diffraction studies were conducted on a Rigaku Nanoviewer (XRF microsource generator, MicroMax 007HF), with a rotating anode coupled to a confocal Max-Flux ${ }^{\circledR}$ Osmic mirror (Applied Rigaku Technologies, Austin, USA) producing beam with a wavelength of $1.5418 \AA$ or $(8 \mathrm{KeV})$. Samples were put in a $2.0 \mathrm{~mm}$ diameter glass capillary (Glaskapillaren GLAS, Glas-Technik \& Konstruktion, Schönwalde-Glien, Germany) with a sample-detector distance of $156 \mathrm{~mm}$. The scans were performed with a transmission geometry setting from $5^{\circ}$ up to $50^{\circ}$. The XRD intensities obtained were plotted against the scattering angle, $2 \Theta$.

\section{RESULTS}

\section{COMPOSITION MAPS AND COLLOIDAL PROPERTIES OF POLYMER PARTICLES}

A general feature of the nanoprecipitation process is that the production of small and monodisperse polymer nanoparticles is restricted to a very narrow region of the phase diagram, the so-called Ouzo domain where the polymer solution is sufficiently diluted and the water fraction high enough to induce supersaturation, resulting in nucleation of small polymer 
particles. $^{26,29}$ The composition map of the PCL45k/THF/water system reflects this typical behavior (Figure 1), in agreement with previous studies on similar systems. ${ }^{16,30}$ At higher PCL concentrations, large-scale fluctuations of the polymer concentration can grow, giving rise to a spinodal decomposition characterized by the formation of large flakes or gel-like sediments depending on the THF/ $\mathrm{H}_{2} \mathrm{O}$ ratio. ${ }^{16}$ While only the left hand boundary of the spinodal region is represented in Figure 1, it is expected that the right hand boundary merges with the binodal at larger polymer concentrations, as computed from the Flory-Stockmayer theory for the PMMA/acetone/water system. ${ }^{16}$ The composition trajectories illustrate the pathway through the phase diagram upon addition of water in the polymer solution prepared at various initial concentrations. Just outside the Ouzo boundaries, samples have a milky appearance due to the coexistence of micro- and nanoparticles, illustrating that transitions observed from a region to another are actually gradual (see red triangles in Figure 1). ${ }^{12,35}$ The same phenomenon has already been observed in the past by different authors ${ }^{16,30}$ in particular in the work of Vitale and Katz, ${ }^{36}$ where they identified a region called "unstable Ouzo" composed of large particles. The term "unstable" in this context is used to describe a very low colloidal stability compared to the Ouzo region, in which particles are kinetically stabilized for longer times. Here, it is noteworthy that the Ouzo region is separated from the binodal miscibility curve by such intermediate phaseseparated domain. It emphasizes that the large polymer structures formed at low level of supersaturation can reorganize into smaller and probably denser ones by increasing the water content. Figure 1 also shows that the supersaturation has to be increased to match the Ouzo conditions when the polymer concentration is increased, in agreement with the observation of Stainmesse et al. on a similar system. ${ }^{30}$ 
The composition maps plotted in Figure 1 reveal that the mixing protocol affects the size of the Ouzo domain, namely the formation of nanoparticles is observed for a larger range of PCL concentrations and THF contents in the case of the FA method compared to the DD one. In other words, the conditions of metastability do not depend solely on the system composition but also on the mixing time, thus indicating the non-equilibrium nature of the process. Moreover, some polymer sedimentation was always observed after a while with the DD method in the Ouzo domain, especially at low THF content. In fact, while more than $90 \%$ by weight of the initial polymer amount was recovered in the supernatants of the formulation prepared by the FA approach, this percentage could drop down to $20 \%$ with the DD method which emphasizes a lower colloidal stability. This is due to the fact that small and large particles are generated through this method and larger ones coalesce more easily (see Table S1 in SI).

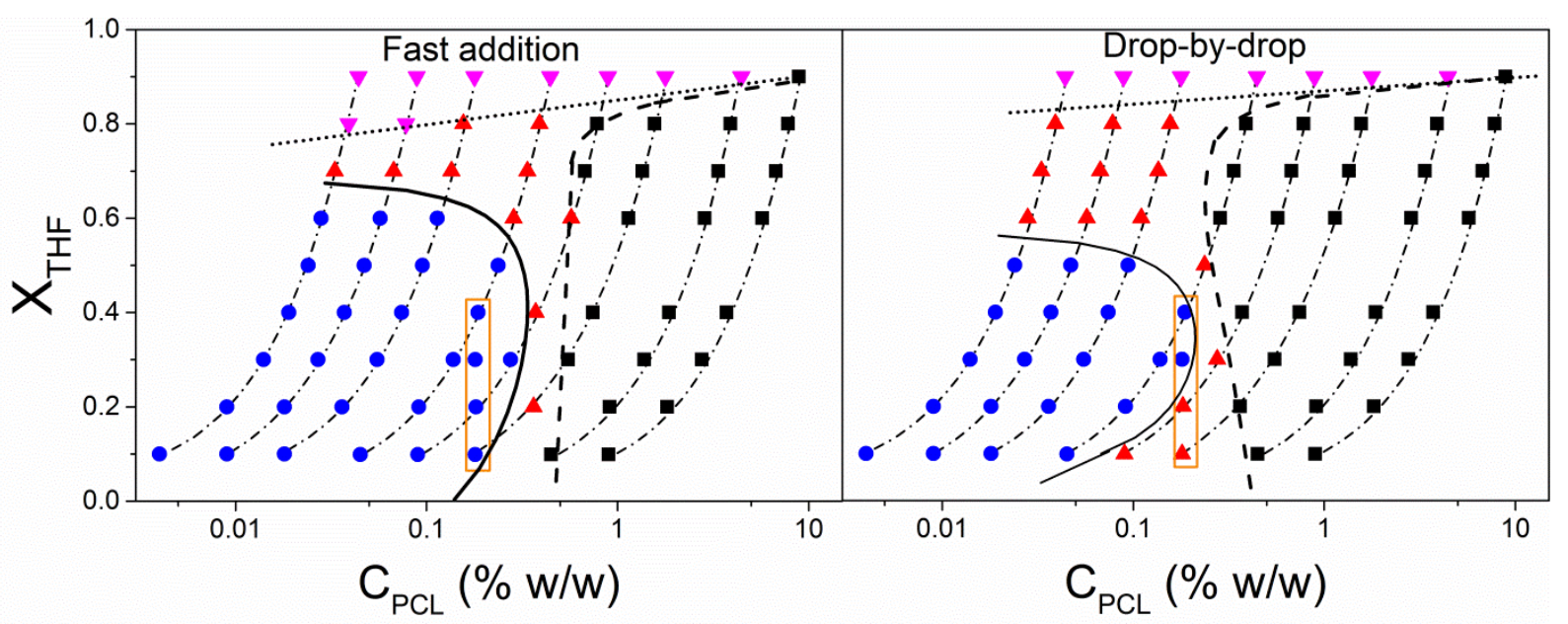

Figure 1. Composition maps of the ternary PCL45k/THF/ $\mathrm{H}_{2} \mathrm{O}$ system obtained by to two water addition pathways, the fast (FA) and the drop-by-drop addition (DD). The THF volume faction $\left(\mathrm{X}_{\mathrm{THF}}\right)$ is reported as a function of the final PCL concentration $(\% \mathrm{w} / \mathrm{w})$. The aspect of each sample was visually inspected after the mixing was completely ended. Four domains were identified: $\nabla$ transparent solutions (apparent polymer solution); - Bluish dispersions 
(nanoparticles); $\boldsymbol{\Delta}$ milky aspect (micro- and nanoparticles); macroscopic separation (flakes or gel-like sediments). The binodal (miscibility-limit, dotted line), Ouzo (straight line) and spinodal (stability-limit, dashed line) boundaries are plotted in bold. The dashed-dotted lines represent the composition trajectories, from high THF contents to lower ones as water is added. The samples selected for further characterization are surrounded by an orange rectangle.

SEM analysis performed on particle dispersions confirms the presence of nanoparticles of about $300 \mathrm{~nm}$ inside the Ouzo regime (Figure 2. $\mathrm{A}, \mathrm{C}_{\mathrm{PCL}}=0.05 \% \mathrm{w} / \mathrm{w}, \mathrm{X}_{\mathrm{THF}}=0.20, \mathrm{FA}$ ). Close to the boundaries of the Ouzo domain, particles of less than $1 \mu \mathrm{m}$ coexist with microparticles (Figure 2.B, $\mathrm{C}_{\mathrm{PCL}}=0.20 \% \mathrm{w} / \mathrm{w}, \mathrm{X}_{\mathrm{THF}}=0.20, \mathrm{FA}$ ) while large aggregates are formed outside (Figure 2.C, $\left.\mathrm{C}_{\mathrm{PCL}}=0.20 \% \mathrm{w} / \mathrm{w}, \mathrm{X}_{\mathrm{THF}}=0.20, \mathrm{DD}\right)$. From the micrographs, it is clear that aggregation or coalescence phenomena occurred during the preparation of SEM samples as these large structures were not detected by DLS in solutions (see below). It also means that the THF content in particles varies according to the area of the composition map so that particles are solid and sticky in the Ouzo domain, while they are rather fluid out of the Ouzo domain or near the borders, coalescing through "diffusion limited coalescence" (DLC) (Figure 2B). Finally, the influence of the mixing method is obvious when comparing two dispersions with the same composition $\left(\mathrm{C}_{\mathrm{PCL}}=0.2 \% \mathrm{w} / \mathrm{w}, \mathrm{X}_{\mathrm{THF}}=0.2\right)$ prepared either through FA (Figure 2.B) or DD (Figure 2.C) pathway. 


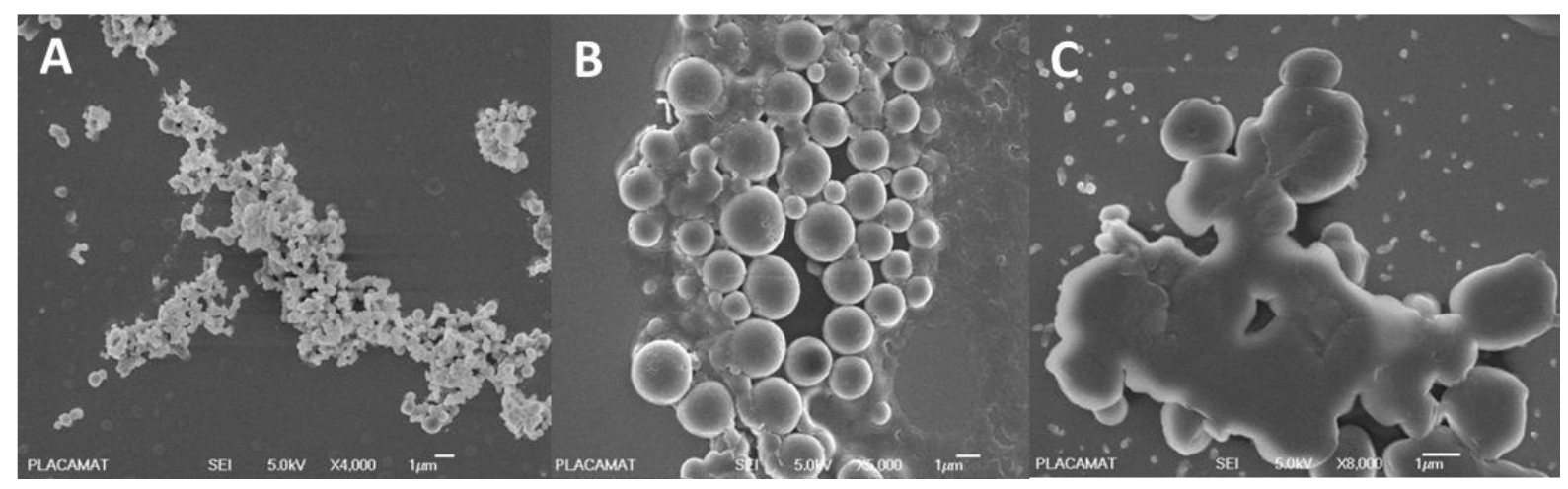

Figure 2. SEM photographs of PCL particles prepared at $\mathrm{X}_{\mathrm{THF}}=0.20$ under various conditions of polymer concentration and mixing method: $\mathrm{C}_{\mathrm{PCL}}=0.05 \% \mathrm{w} / \mathrm{w}$, fast addition $(\mathrm{A}), \mathrm{C}_{\mathrm{PCL}}=0.20 \%$ $\mathrm{w} / \mathrm{w}$, fast addition $(\mathrm{B})$ and $\mathrm{C}_{\mathrm{PCL}}=0.20 \% \mathrm{w} / \mathrm{w}$, drop-by-drop addition $(\mathrm{C})$.

The colloidal state of four systems with the same final PCL content $(0.18 \% \mathrm{w} / \mathrm{w})$ but THF fractions varying from 0.1 to 0.4 was assessed by dynamic light scattering (DLS) according to the FA and DD mixing methods. The selected compositions are highlighted in Figure 1. For the FA approach, the four compositions are well inside the Ouzo domain while for the DD approach they are close to the boundary $\left(\mathrm{X}_{\mathrm{THF}}=0.4\right.$ and 0.3$)$ or slightly out the Ouzo domain $\left(\mathrm{X}_{\mathrm{THF}}=0.2\right.$ and 0.1). DLS clearly evidences the effect of the formulation pathway on the mean hydrodynamic size and polydispersity of PCL particles. For the FA procedure, relatively small particles with a narrow distribution are obtained whatever the $\mathrm{THF} / \mathrm{H}_{2} \mathrm{O}$ ratio considered while for the DD protocol, small particles around $100 \mathrm{~nm}$ coexist with much larger ones (Figure 3.A). 

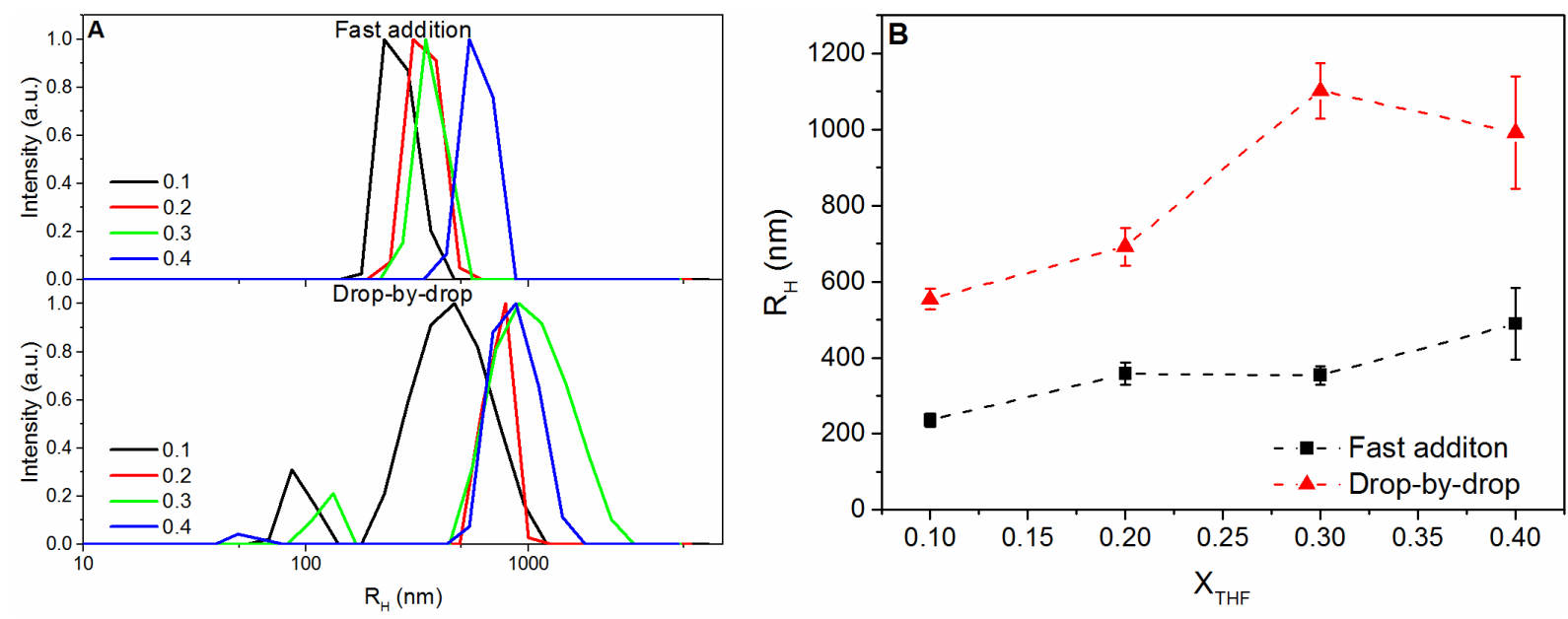

Figure 3. A) Intensity-average size distribution of PCL $45 \mathrm{kDa}$ particles prepared by fast addition or drop-by-drop injection at different THF volume fractions $\left(\mathrm{X}_{\mathrm{THF}}\right)$ : 0.1 (black); 0.2 (red); 0.3 (green); 0.4 (blue). B) Mean hydrodynamic radius of PCL $45 \mathrm{kDa}$ particles are reported as a function of $\mathrm{X}_{\mathrm{THF}}$. The final PCL content is $0.18 \% \mathrm{w} / \mathrm{w}$. DLS analyses were performed right after preparation.

Therefore, DLS confirms the trend depicted in the composition maps, namely a short mixing time favors a homogeneous supersaturation of the polymer in bad solvent with the subsequent formation of small and uniform nuclei and eventually the generation of nanoparticles with a relatively narrow size distribution. Figure 3.B put in evidence a gradual increase of the particle size with the $\mathrm{THF}$ volume ratio $\left(\mathrm{X}_{\mathrm{THF}}\right)$. In high supersaturation conditions (small $\left.\mathrm{X}_{\mathrm{THF}}\right)$ the nanoprecipitation process is dominated by a high nucleation rate which ultimately results in smaller particles. In low supersaturation condition (high $\mathrm{X}_{\mathrm{THF}}$ ), nucleation events are more rare since the nucleation barrier is high. Consequently, nuclei are less numerous and they can grow faster than they nucleate which result in polymer particles of larger sizes. A similar behavior was observed with a PCL of lower molecular weight (see Figure S3). Moreover, the particle size 
increase seen at higher $\mathrm{X}_{\mathrm{THF}}$ ratios could be attributed to some extent to the particle swelling by $\mathrm{THF}^{16}$

Particles stability is known to be directly related to presence of surface charge, which in turn depends on the presence of charged or ionizable groups on the polymer backbone or chainends. ${ }^{38}$ Zeta potentials measured in solvent/water mixtures at $\mathrm{X}_{\mathrm{THF}}=0.1$ were $-55.8 \pm 0.8 \mathrm{mV}$ for particles prepared by FA and $-28 \pm 8 \mathrm{mV}$ for those prepared by DD. The negative values of zeta potential arise from the presence of PCL terminal carboxylic groups segregating at the particle surface. ${ }^{13,27,39}$ At higher THF content, however, measurements were not reliable, probably because the carboxylic groups on PCL are less dissociated. While particles have rather high absolute values of zeta potential, they are not very stable in $\mathrm{THF} / \mathrm{H}_{2} \mathrm{O}$ mixtures as shown by the size variation of particles in aging dispersions. Figure 4 illustrates that the aging process occurs more quickly for the particles prepared by DD procedure, probably because of their larger initial sizes. ${ }^{40}$ In fact, after 11 hours, only the smaller particles were detected by DLS, whereas the larger particles aggregated and sedimented. For FA instead, an appreciable decrease in $R_{H}$ is observed only after 24 hours. Sedimentation is still noticeable but to a lesser extent. As further proof of the fact that FA particles are more stable, the scattering intensity is almost constant over 24 hours, whereas in the DD system the intensity drops off after 11 hours (see Figure S4). The increase in size of the particles can be either due to Ostwald ripening or coalescence in presence of THF, as reported previously. ${ }^{41}$ 


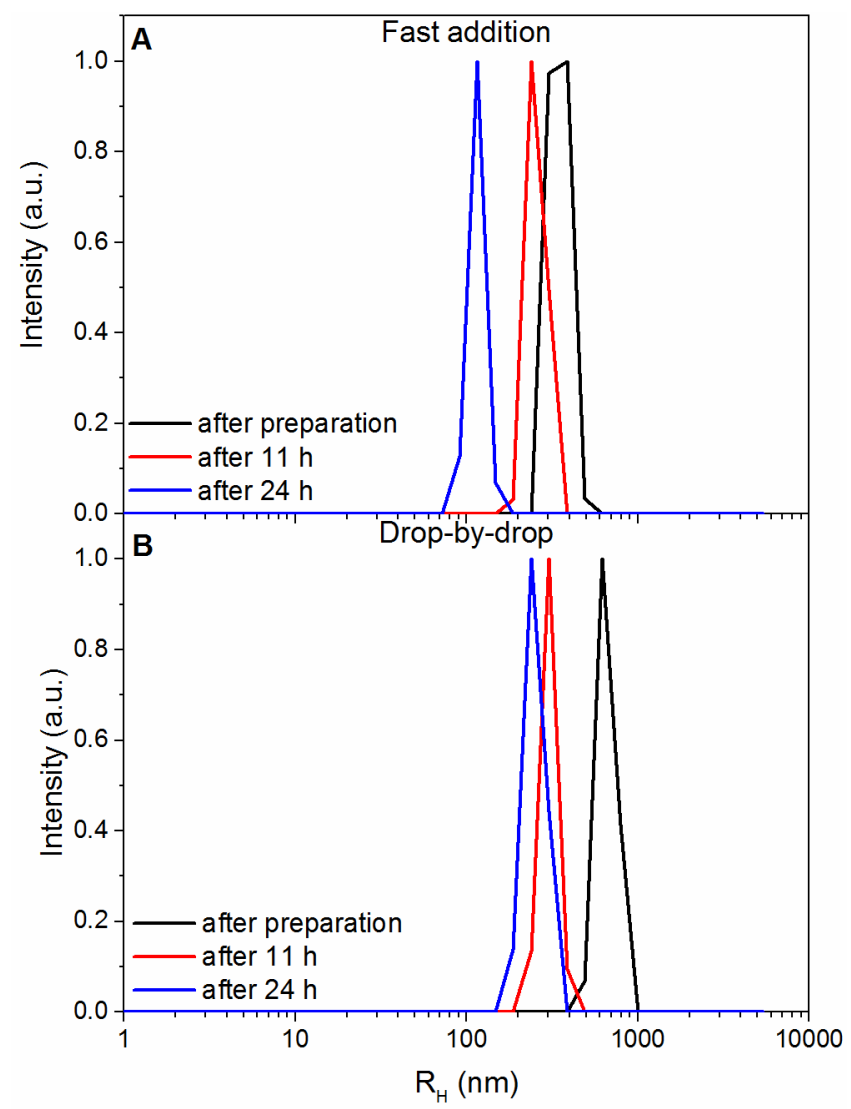

Figure 4. Intensity-average size distribution of PCL $45 \mathrm{kDa}$ particles prepared by fast addition or drop-by-drop injection, with $\mathrm{X}_{\mathrm{THF}}=0.2$ in both cases, and analyzed after preparation (black line), $11 \mathrm{~h}$ (red line) and $24 \mathrm{~h}$ (blue line). The final PCL content is $0.18 \% \mathrm{w} / \mathrm{w}$.

\section{CRYSTALLINE PROPERTIES OF POLYMER PARTICLES}

\section{$\mu D S C$ analysis of PCL particles prepared under various conditions}

While the mixing conditions affect the nanoparticle size, polydispersity and stability, it is also of interest to investigate their role on the crystallinity properties of the polymer particles. PCL of $45 \mathrm{kDa}$ and $2 \mathrm{kDa}$ having melting temperatures, $\mathrm{T}_{\mathrm{m}}$, equal to $58^{\circ} \mathrm{C}$ and $51{ }^{\circ} \mathrm{C}$ and crystallinity degrees, $\mathrm{X}_{c}$, equal to $52 \%$ and $57 \%$, respectively, have been used to investigate this aspect (see 
Figure $\mathrm{S} 1)$. The four selected compositions surrounded by an orange rectangle in Figure 1 ( $\mathrm{X}_{\mathrm{THF}}$ $=0.1-0.2-0.3-0.4)$ as well as $\mathrm{X}_{\mathrm{THF}}=0.15$ have been analyzed by $\mu \mathrm{DSC}$ right after the nanoprecipitation, without removing the THF from the medium. Figure 5 shows the thermograms of PCL $45 \mathrm{kDa}$ particles prepared by FA and DD approach at various $\mathrm{X}_{\mathrm{THF}}$ (the results for PCL $2 \mathrm{kDa}$ are reported in Figure S5). Similar results were obtained after the first and second scans from 10 to $50^{\circ} \mathrm{C}$ at $1{ }^{\circ} \mathrm{C} / \mathrm{min}$ (Figure $\mathrm{S} 6$ ).

Three main observations can be drawn from Figure 5: i) the strong decrease of the melting temperatures of the particles prepared through the FA mixing method, from $42^{\circ} \mathrm{C}$ to $21^{\circ} \mathrm{C}$ when $\mathrm{X}_{\mathrm{THF}}$ is increased from 0.1 to 0.4 ; ii) a similar trend for the DD mixing method but with illdefined DSC traces at $\mathrm{X}_{\mathrm{THF}}<0.30$; iii) the presence of two endotherms in most of the thermograms.

The observed decrease of $T_{m}$ is related to the presence of increasing amount of THF in the nanoparticles which interferes with the crystallization process, resulting likely in crystalline structures composed of thinner lamellae associated with lower melting points. For the same reasons, the degree of crystallinity of the polymer in NPs is also lowered as compared to the starting PCL material (Figure 6.B). 


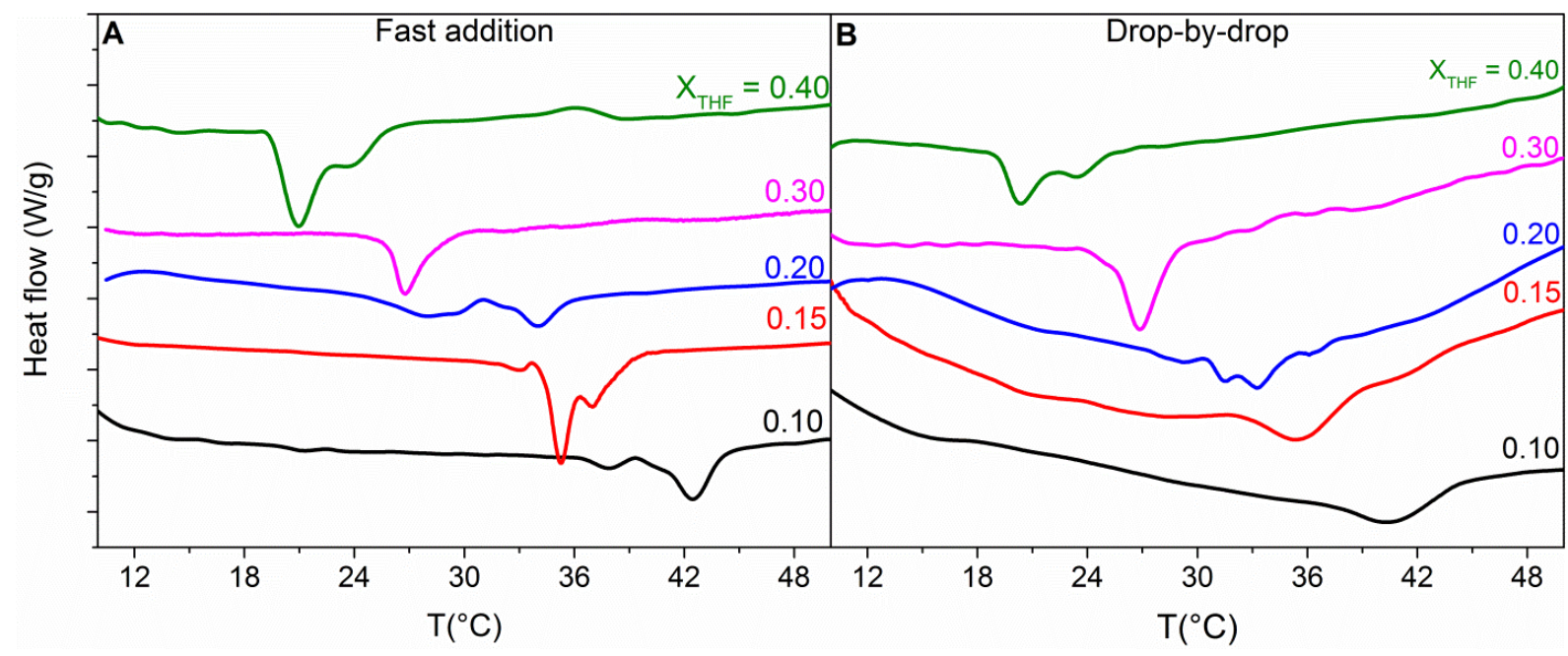

Figure 5. $\mu \mathrm{DSC}$ curves for PCL $45 \mathrm{kDa}$ particles prepared by fast addition (A) and drop-by-drop (B) at different THF volume fractions, $\mathrm{X}_{\mathrm{THF}}$. The final PCL content is $0.18 \% \mathrm{w} / \mathrm{w}$ but owing to some sedimentation, especially with the DD approach, the solid content was systematically determined by thermogravimetric analysis. The data correspond to a first scan from 10 to $50^{\circ} \mathrm{C}$ at $1{ }^{\circ} \mathrm{C} / \mathrm{min}$. The curves are shifted in the y axis in order to improve the clarity of the graph.

This behavior is in good agreement with the melting point depression in polymer-diluent blends described by Flory. ${ }^{42}$ When a semi-crystalline polymer above its melting point is diluted with a miscible low-molecular weight component and cooled to a temperature where crystallization occurs, the measured melting point upon heating is lower than $T_{m}$ of the pure polymer. ${ }^{43}$ While the nanoprecipitation corresponds to an anti-solvent crystallization rather than a cooling crystallization, the melting point depression theory seems to adequately describe experimental results obtained herein. In order to apply this theory, the volume fraction of diluent which corresponds here to the amount of THF entrapped within particles must be known. This aspect will be detailed later in the text. Besides, the plasticization effect of PCL by water can be ruled out since only a small decrease of the $\mathrm{T}_{\mathrm{m}}$ was observed when PCL is in pure water. 
Conversely, pure THF has a dramatic effect on $\mathrm{T}_{\mathrm{m}}$ as predicted by Flory's theory (see Figure $\mathrm{S} 7$ and $\mathrm{S} 8$ in $\mathrm{SI})$.
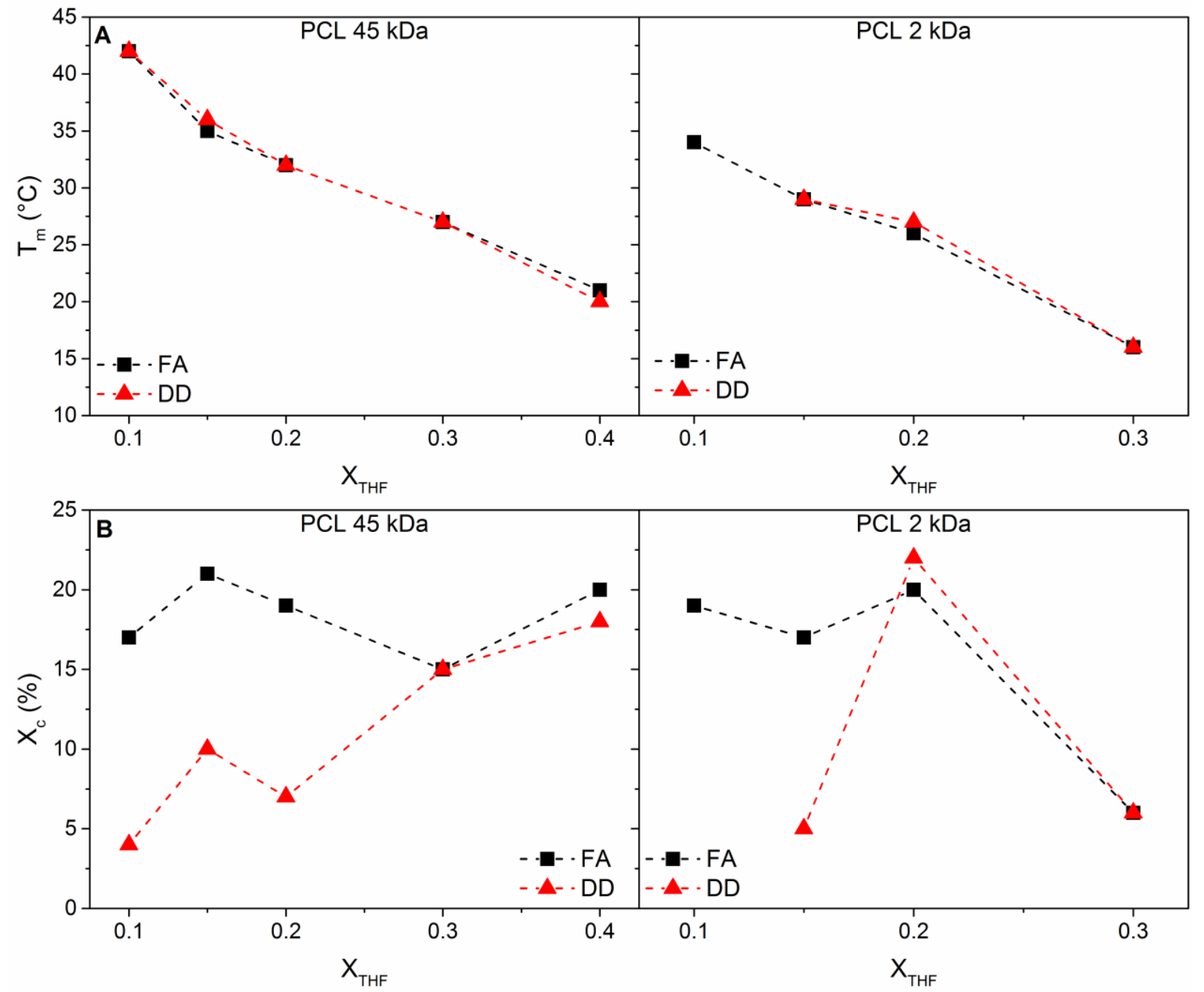

Figure 6. Melting temperatures, $T_{m}(A)$ and degrees of crystallinity, $X_{c},(B)$ for PCL 45 and 2 $\mathrm{kDa}$ particles prepared by nanoprecipitation from a THF solution through fast (FA, black squares) and drop-by-drop (DD, red triangles) addition of water. All THF compositions are in the Ouzo domain for FA addition. For DD addition sample at $\mathrm{X}_{\mathrm{THF}}<0.3$ are outside the Ouzo boundary. For PCL $2 \mathrm{k}$ no precipitation is observed for $\mathrm{X}_{\mathrm{THF}}>0.3$. 
According to the values of $\mathrm{T}_{\mathrm{m}}$ obtained for both PCL $45 \mathrm{k}$ and PCL $2 \mathrm{k}$ at various $\mathrm{X}_{\mathrm{THF}}$, there is no impact at first glance of the formulation pathway (FA and DD) on the melting of PCL particles (Figure 6.A). In other words, the melting temperature seems to solely depend on the final volume fraction of THF used for the nanoprecipitation and not on the mixing method. In a similar manner, the crystalline content $\left(\mathrm{X}_{\mathrm{C}}\right)$ is constant around $20 \%$ for both PCL molecular weights whatever the THF content and the mixing method, except for the DD approach when $\mathrm{X}_{\mathrm{THF}}<0.30(\mathrm{PCL} 45 \mathrm{k})$ or $\mathrm{X}_{\mathrm{THF}}<0.20(\mathrm{PCL} 2 \mathrm{k})$ (Figure 6B). Under these conditions, the endotherms are barely detected on thermograms thus demonstrating that the particles formed in the "unstable Ouzo" domain have lower polymer crystallinity (Figure 5.B). For PCL 2k, the polymer does not precipitate when $\mathrm{X}_{\mathrm{THF}} \geq 0.40$ because of its higher solubility in $\mathrm{THF} / \mathrm{H}_{2} \mathrm{O}$ mixtures compared to PCL 45k. For the same reason, the crystalline content is quite low for PCL $2 \mathrm{k}$ at $\mathrm{X}_{\mathrm{THF}}=0.30$.

Another peculiar aspect of the thermograms shown in Figure 5 is that, the melting peaks are often split into two contributions. It has been reported in literature that this can be due to different orientations of the crystalline structure and/or to crystalline lamellae with different fold numbers. ${ }^{23}$ PCL has an orthorhombic unit cell, ${ }^{44,45}$ with an XRD spectrum characterized by three strong reflections at $2 \Theta$ around $21.4^{\circ}, 22^{\circ}$ and $23.7^{\circ}$, corresponding to the (110), (111) and (200) planes of the orthorhombic crystal structure respectively. ${ }^{46}$ As one can see from Figure 7, PCL particles display the same XRD pattern. Despite the lower intensity due to a slightly lower crystallinity degree, the peaks are well-defined and sharp. Hence, there is no significant difference in the orientation or folding number compared to the bulk polymer. Moreover, in order to avoid any dependence on the pre-treatment history, we compared two procedures commonly used for this purpose: freeze drying and particles centrifugation. The results are not 
preparation-dependent. However, the pellets separated by centrifugation have a lower intensity because the solvent is still trapped inside the particles, in agreement with the observations previously ruled out. In light of these results, the broadening of the melting transition observed by $\mu \mathrm{DSC}$ can rather be explained in terms of size polydispersity of crystalline lamellae and/or variations of THF content inside the nanoparticles.

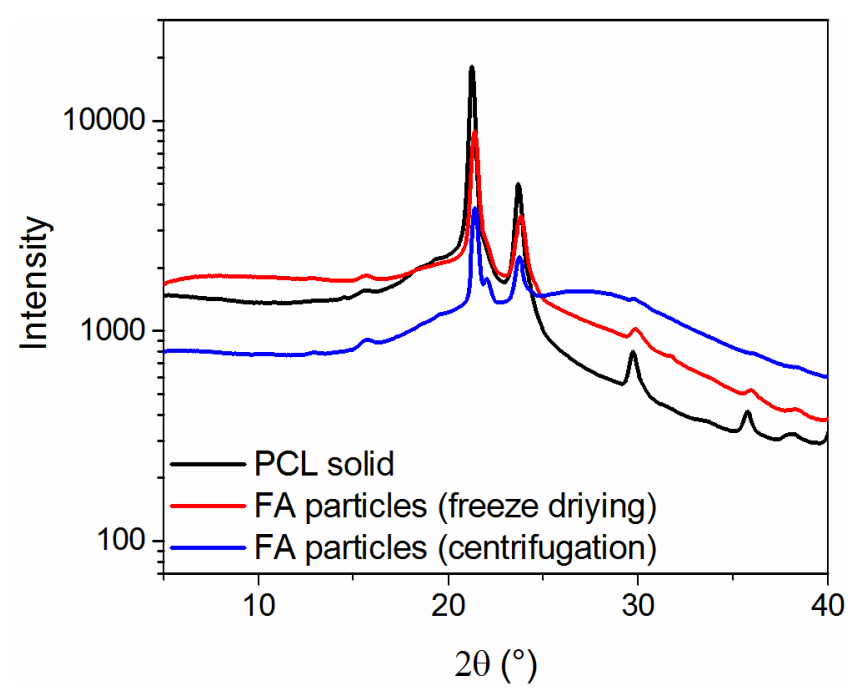

Figure 7. XRD patterns of the solid PCL (black line) and PCL $45 \mathrm{kDa}$ particles at $\mathrm{X}_{\mathrm{THF}}=0.2$ prepared by fast addition after freeze drying (red line) or centrifugation (blue line).

\section{THF fraction in PCL nanoparticles}

Starting from PCL solutions in pure THF, the nanoprecipitation process leads to a solvent partition between nanoparticles and water which is responsible of the depression of the PCL melting point (Table 1). Two methods based on ${ }^{1} \mathrm{H}$ NMR spectroscopy and contrast variation by SANS have been set up to determine the THF content and its distribution in nanoparticles without altering the solvent partition. In the first method, the particles were collected by centrifugation and dissolved in deuterated chloroform which is a good solvent of PCL and 
miscible with THF as demonstrated by the high resolution of NMR spectra (see Figure S2). The volume fraction of THF in nanoparticles $\left(\phi_{\text {ТнF }}\right)$ was derived from the integral ratio of peaks of PCL and THF. For the FA approach, $\phi_{\mathrm{THF}}$ regularly increases with the initial THF content (Table 1). Mean values of the partition coefficient (K) are 1.05 for PCL $45 \mathrm{kDa}$ and 2.5 for PCL $2 \mathrm{kDa}$ in agreement with a high affinity of THF for PCL, especially for the low molecular weight one. For the DD method, $\phi_{\text {THF }}$ values are similar to those obtained by FA when the composition matches the Ouzo domain $\left(\mathrm{X}_{\mathrm{THF}}=0.30\right.$ for PCL $45 \mathrm{k}$ and $\mathrm{X}_{\mathrm{THF}}=0.20$ for PCL $\left.2 \mathrm{k}\right)$. Out of the Ouzo domain, the THF content is much higher in agreement with the formation of larger and probably less dense particles in this area of the phase diagram.

Table 1. Volume fraction of ( $\left.\phi_{\mathrm{THF}}\right)$ trapped inside PCL $45 \mathrm{kDa}$ and $2 \mathrm{kDa}$ particles prepared by fast addition and drop-by-drop addition at different $\mathrm{X}_{\mathrm{THF}}$ determined by NMR.

\begin{tabular}{ccc|ccc} 
PCL 45 kDa & \multicolumn{2}{c|}{$\phi_{\text {THF }}$} & PCL 2 kDa & \multicolumn{2}{c}{$\phi_{\text {THF }}$} \\
\hline$X_{\text {THF }}$ & FA & DD & $X_{\text {THF }}$ & FA & DD \\
\hline 0.10 & $0.14 \pm 0.05$ & $0.35 \pm 0.06$ & 0.10 & $0.16 \pm 0.03$ & $0.66 \pm 0.09$ \\
0.20 & $0.22 \pm 0.05$ & $0.59 \pm 0.04$ & 0.20 & $0.35 \pm 0.08$ & $0.39 \pm 0.03$ \\
0.30 & $0.35 \pm 0.06$ & $0.41 \pm 0.12$ & & & \\
& & & & &
\end{tabular}

In addition to the NMR, the contrast variation method by SANS was used as an original way to determine at the same time the THF content and distribution within PCL nanoparticles (Figure 8). This procedure is relevant for multicomponent systems to highlight one component at a time and get more detailed information about the system. ${ }^{47}$ We decided to focus on PCL $45 \mathrm{kDa}$ particles prepared at $\mathrm{X}_{\mathrm{THF}}=0.20$ using both formulation pathways since it is the best compromise in terms of scattering intensity and difference in crystallinity degree. At 
intermediate $\mathrm{q}$, the scattered intensity follows a $\mathrm{q}^{-4}$ dependence due to the presence of smooth and dense spherical objects (Porod law). The same behavior is observed for all the conditions, even when the PCL scattering length density is matched by the solvent (THF-d/ $\mathrm{H}_{2} \mathrm{O}=20 / 80$ ), i.e. when its contribution is switched off. Then, the scattered intensity arises only from the THF trapped inside the particles. The fact that the scattered intensity trend does not depend on the contrast conditions for both FA and DD methods also demonstrates that the organic solvent is evenly distributed inside the particle. Indeed, if the THF would have been located in a specific region of the particles (near the solution/polymer interface, in the particle core or in small globules, for example), the curves should have displayed a different profile. ${ }^{48}$

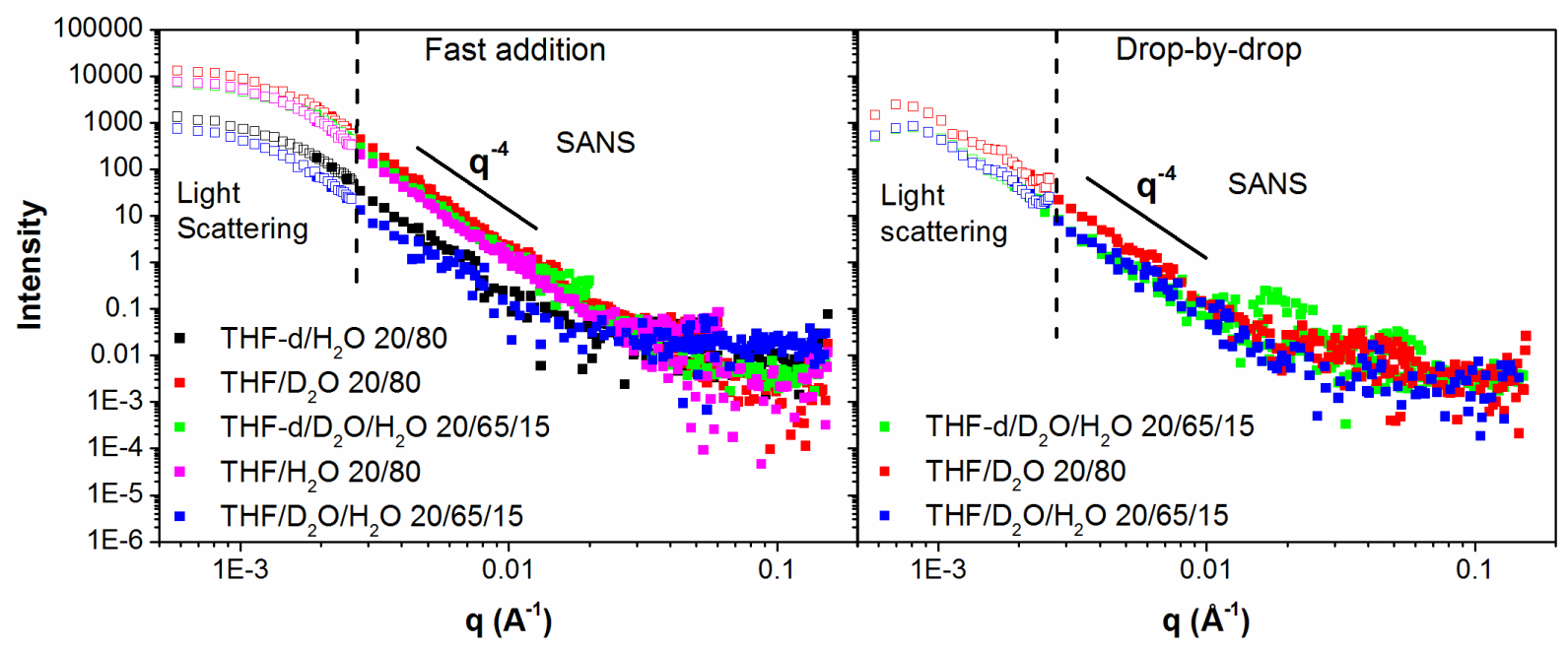

Figure 8. SLS (open symbols) and SANS (filled symbols) intensities vs $q$ at different solvent contrast for PCL $45 \mathrm{kDa}$ particles at $\mathrm{X}_{\mathrm{THF}}=0.20$ prepared by fast addition (A) and drop-by-drop injection (B). SLS curves have been shifted to match SANS curves intensities in the overlapping q-region. 
Then, by considering that the scattered intensity is proportional to $\left(\rho_{\text {particle }}-\rho_{\text {solvent }}\right)^{2}$ where $\rho_{\text {particle }}=\left(1-\phi_{\mathrm{THF}}\right)^{*} \rho_{\mathrm{PCL}}+\rho_{\mathrm{THF}} * \phi_{\mathrm{THF}}$, one can find through a least-squares fitting the $\phi_{\mathrm{THF}}$ value that matches at best the normalized scattered intensity obtained under various contrast conditions. For the fast addition, the volume fraction of THF trapped in particles was found to be $0.15 \pm 0.02$ in quite good agreement with the NMR result. Unfortunately, for the drop-by-drop we could not get an accurate value $(0.35 \pm 0.10)$ because of the inherent polydispersity of the sample and the fast sedimentation of the generated particles (the NPs volume fraction is not constant throughout the measurement).

Experimental $\phi_{\mathrm{THF}}$ values can be compared to the theoretical ones deduced from $\mathrm{T}_{\mathrm{m}}$ values (Figure 6) by applying the theory of the melting point depression proposed by Flory which accounts for the decrease of the melting temperature in semi-crystalline polymers when a diluent is added. According to Flory: ${ }^{42}$

$$
\frac{1}{\mathrm{~T}_{\mathrm{m}}}-\frac{1}{\mathrm{~T}_{\mathrm{m}}^{0}}=\frac{\mathrm{R}}{\Delta \mathrm{H}_{\mathrm{u}}} \frac{\mathrm{v}_{\mathrm{u}}}{\mathrm{v}_{1}}\left(v_{1}-\chi_{1} v_{1}^{2}\right)
$$

where $\mathrm{T}_{\mathrm{m}}^{0}$ is the equilibrium melting point for crystals of infinite MW chains (340 K for PCL) ${ }^{49}$, $\Delta \mathrm{H}_{\mathrm{u}}$ the heat of fusion per mole of repeating unit $(15.9 \mathrm{~kJ} / \mathrm{mol} \text { for PCL })^{33}, \mathrm{v}_{\mathrm{u}} / \mathrm{v}_{1}$ the ratio of the molar volume of this unit and those of the diluent, $v_{1}$ the volume fraction of diluent, i.e $v_{1}$ corresponds to $\phi_{\mathrm{THF}}$ trapped inside the core of the polymer particles in our case, and $\chi_{1}$ the interaction parameter determined from the relationship $\chi_{1}=0.34+\left(\frac{\mathrm{v}_{1}}{\mathrm{RT}}\right)\left(\delta_{1}-\delta_{\mathrm{u}}\right)^{2}$ where $\delta_{\mathrm{i}}$ are Hildebrand solubility parameters. ${ }^{50}$ The Flory relationship makes the assumption that solvent molecules are completely rejected from the crystalline phase. Values of $\phi_{\mathrm{THF}}$ derived from Flory's equation for PCL 45k together with those determined by NMR are plotted as function of $\mathrm{X}_{\mathrm{THF}}$ in Figure 9. When the compositions are in the Ouzo domain, $\phi_{\mathrm{THF}}$ values determined by 
NMR are lower than expected from Flory's theory. In other words, the melting point of polymer nanoparticles is smaller than what the Flory's theory predicts from the actual THF volume content. Conversely there is a good agreement between actual and predicted $\phi_{\mathrm{THF}}$ values for the DD addition at low $\mathrm{X}_{\mathrm{THF}}(<0.30)$ where the system is slightly out of the Ouzo domain. It is worth mentioning here that small variations on $\chi_{1}$ do not have dramatic consequences on the $\phi_{\mathrm{THF}}$ calculated using Flory relationship, corroborating the reliability of the fit and the resulting considerations.

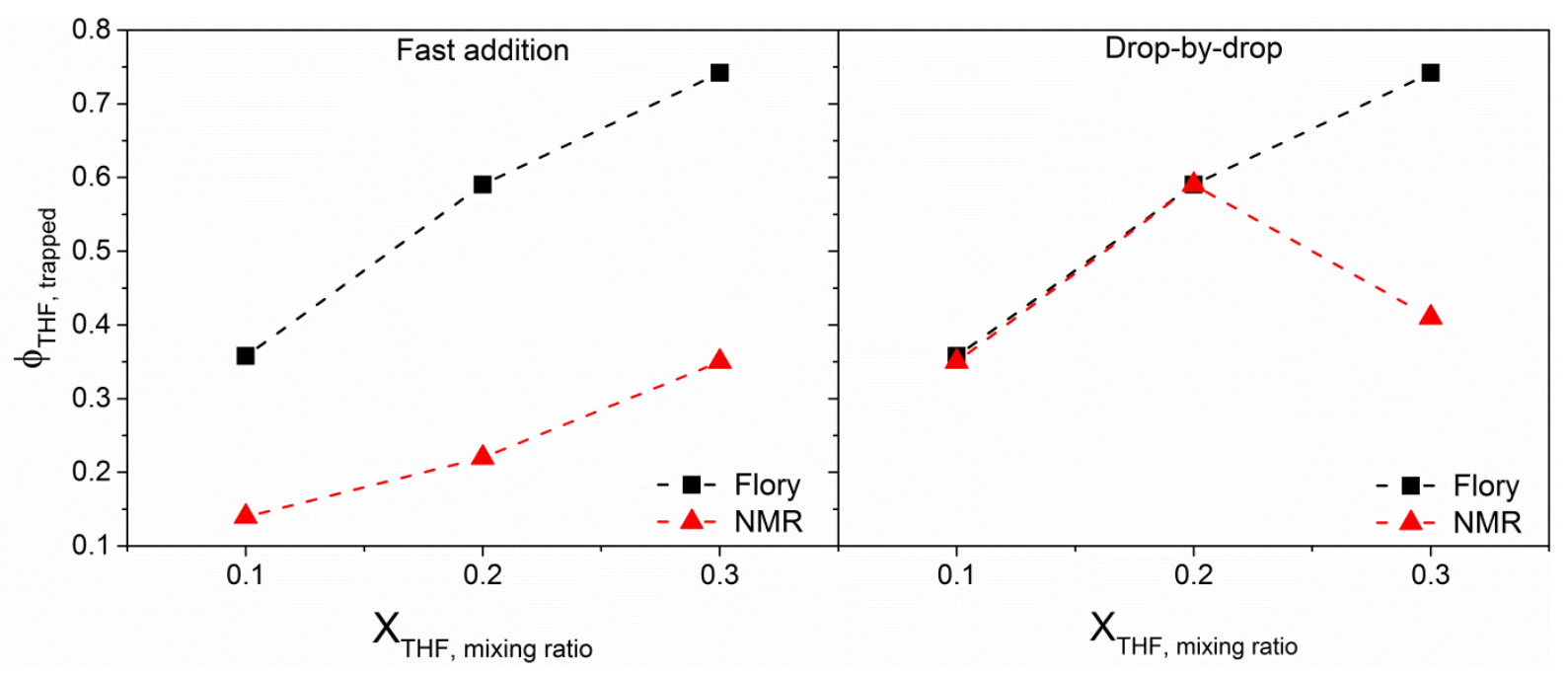

Figure 9. THF volume fraction, $\phi_{\mathrm{THF}}$, entrapped in the nanoparticles determined from $T_{m}$ values by applying the Flory melting point depression theory (black squares) and NMR analysis (red triangles) for PCL 45k for both fast addition and drop-by-drop mixing methods at various $\mathrm{X}_{\mathrm{THF}}$.

Finally, the organic solvent was removed by dialysis from the PCL $45 \mathrm{kDa}$ particles prepared in the Ouzo domain at $\mathrm{THF} / \mathrm{H}_{2} \mathrm{O}=0.20$ by FA and DD. There is a good recovery of the crystalline properties of PCL upon THF removal even for particles prepared by the DD method where the degree of crystallinity is doubled after dialysis (Table 2). This demonstrates that 
polymer chains are not in a frozen state in particles and can still reorganize under appropriate conditions. Regarding the size distribution, no difference was detected before and after dialysis for the particles prepared by fast addition, whereas for the drop-by-drop it is possible to observe two populations, especially one of larger size, confirming the poor colloidal stability of the DD system (Figure 10).

Table 2. Melting temperature $\left(\mathrm{T}_{\mathrm{m}}\right)$ and degree of crystallinity $\left(\mathrm{X}_{\mathrm{c}}\right)$ for PCL $45 \mathrm{kDa}$ particles at $\mathrm{X}_{\mathrm{THF}}=0.20$ prepared by fast addition and drop-by-drop before and after dialysis.

\begin{tabular}{ccc|ccc} 
Fast addition & $\mathrm{T}_{\mathrm{m}}\left({ }^{\circ} \mathrm{C}\right)$ & $\mathrm{X}_{\mathrm{c}}(\%)$ & Drop-by-drop & $\mathrm{T}_{\mathrm{m}}\left({ }^{\circ} \mathrm{C}\right)$ & $\mathrm{X}_{\mathrm{c}}(\%)$ \\
\hline Before dialysis & 32 & 19 & Before dialysis & 32 & 7 \\
After dialysis & 58 & 42 & After dialysis & 52 & 14
\end{tabular}

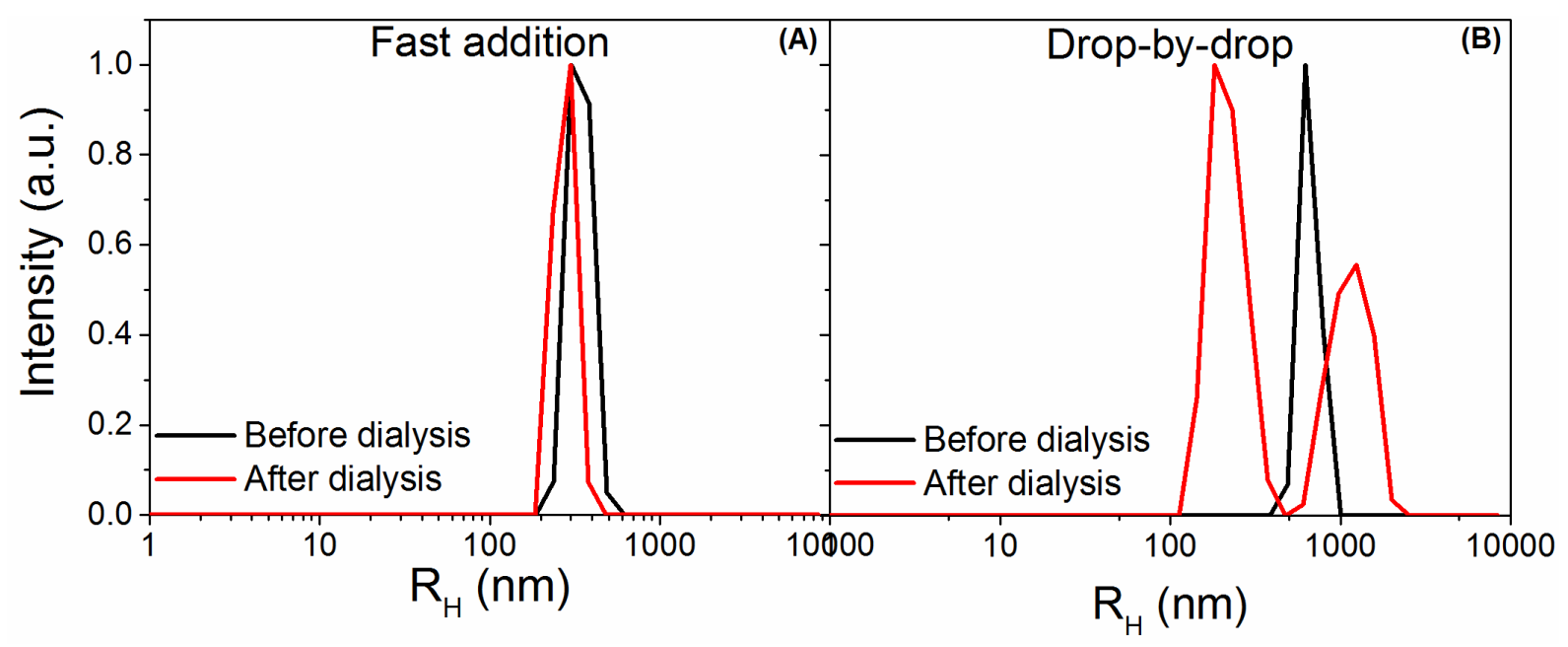

Figure 10. Intensity-averaged distribution of the hydrodynamic radius $\left(\mathrm{R}_{\mathrm{H}}\right)$ of PCL $45 \mathrm{kDa}$ particles at $\mathrm{THF} / \mathrm{H}_{2} \mathrm{O}=0.2$ prepared by fast addition (A) and drop-by-drop injection (B) before (black line) and after dialysis (red line) 


\section{DISCUSSION}

The nanoprecipitation of PCL from polymer solutions in THF has been studied at various polymer concentrations and $\mathrm{THF} / \mathrm{H}_{2} \mathrm{O}$ ratios. Even without any added surfactants the formation of small PCL nanoparticles can be achieved at low polymer concentration when the water content is high enough, that is, under high polymer supersaturation. The particles are stabilized by carboxylic end groups giving rise to a negative zeta potential in $\mathrm{THF} / \mathrm{H}_{2} \mathrm{O}$ mixtures. The socalled Ouzo domain corresponding to the region of formation of nanoparticles has distinct frontiers different from the binodal (miscibility-limit) and spinodal (stability-limit) curves. As a matter of fact, the solubility domain is separated from the Ouzo domain by an intermediate region, the "unstable Ouzo" region, characterized by highly turbid polymer dispersions (Figure 1). Concerning the mechanism of particle formation in the Ouzo domain, the nucleation of nanoparticles driven by the supersaturated state of the polymer is likely the dominant process. Nuclei can then growth either through diffusion of polymer molecules from solution at relatively high THF content (nucleation-growth mechanism) or through aggregation at very low THF fractions, that is, in the high supersaturation regime (nucleation-aggregation mechanism). In the "unstable Ouzo" domain, the formation of micro-sized particles may result either from a limited spinodal decomposition relative to the low polymer concentration, or from a nucleation mechanism where the nuclei are unstable and aggregate. The fact that these microparticles can rearrange into smaller ones at increasing levels of supersaturation shows that polymer chains can be redistributed between the aggregates and the solution. This suggests that the "unstable Ouzo" region corresponds to a pseudo chemical equilibrium state.

The dependence of the solvent displacement method to initial conditions was well evidenced by varying the mixing time between two extremes, the FA and DD formulation pathways, which 
give rise to two distinct composition maps with shifted Ouzo boundaries (Figure 1). Namely a fast mixing time accounts for a larger range of polymer concentrations and THF contents that can be used to obtain stable nanoparticles. The effect of the mixing time can be rationalized by considering the Damkohler number, $D a=\tau_{\text {mix }} / \tau_{\text {precip }}$, where $\tau_{\text {mix }}$ is the mixing time and $\tau_{\text {precip }}$ the overall precipitation time which is composed of the nucleation and the growth (or aggregation) time. ${ }^{15}$ For the FA approach, one may assume that the total volume of water was added into the PCL solution within a few hundred milliseconds. Considering a precipitation time of a few tenths of milliseconds as reported by others ${ }^{17}$ the $D a$ number is likely below 10 , a value that is low enough to ensure a fast and homogeneous supersaturation leading to high nucleation rate and eventually the formation of polymer nanoparticles as shown by SEM and DLS analysis (Figure 2 and Figure 3). The radius of the particles is between 200 and $400 \mathrm{~nm}$ which can be considered a relatively small size since no surfactant has been used. Regarding the DD approach, the mixing time is in the order of tens of seconds, so that the $D a$ number has no physical meaning in this case. The DD method can be rather viewed as a titration approach where water droplets are mixed with the polymer solution at each step of the addition. Then, the system experiences a gradual decrease in the solubility of PCL chains according to the composition trajectories represented in the composition maps (Figure 1). It is important to note that trajectories systematically cross the "unstable Ouzo" domain at low levels of supersaturation before reaching the stable Ouzo region. The presence of polymer microparticles must then favor heterogeneous nucleation at higher supersaturation, thus giving rise to larger particles than those obtained when the water is quickly brought inside the Ouzo domain through the FA method. For same reason the Ouzo domain corresponding to the DD method is restricted to a lower concentration range and THF content. Within the Ouzo domain regardless of the mixing method, the particle size is 
systematically increased at higher THF contents (Figure 3.B) as the particle growth is favored over the nucleation step under such conditions. When the system composition is slightly outside the Ouzo domain, the particles are unstable and can aggregate or coalesce depending on the THF fraction (Figure 4). DLS analysis showed that a mixture of small and large particles is obtained in this area of the composition map (Figure 3.A).

Particles obtained in the Ouzo domain by FA or DD method exhibit semi-crystalline properties evidenced by $\mu \mathrm{DSC}$ and $\mathrm{XRD}$ analysis of the particle dispersions in various $\mathrm{THF} /$ water mixtures. When the THF fraction in the nanoprecipitation medium is increased, the melting temperature is decreased and the melting range is broadened but the crystalline phase remains pure (Figure 6 and 7). This is in agreement with the presence of increasing amounts of THF evenly distributed within particles but excluded from the crystalline lattice. The melting point depression theory of Flory for a crystalline polymer-diluent system adequately describes this behaviour even though $T_{m}$ values are smaller than what the Flory's theory predicts from the actual THF volume content in particles. The origin of this difference might be related to the fact that the crystallization is obtained here through an anti-solvent process rather than a cooling process from the melt. Importantly, there is no significant difference of $\mathrm{T}_{\mathrm{m}}$ between the FA and DD method thus emphasizing the same lamellar thickness of crystallites under both mixing conditions. ${ }^{51}$ In addition, the crystallization degree is almost constant in the Ouzo domain, around 20\%, whatever the conditions of mixing and THF contents (Figure 6). At first glance it could have been expected that a slow change in solvent quality for the DD approach would favor the polymer crystallization over fast mixing as observed for a slow cooling rate in melt crystallization. Rather, it suggests that the formation of crystalline domains in nanoparticles is a result of thermodynamic factors rather than kinetic ones. Namely, the polymer crystallization 
probably occurs at longer times than the polymer precipitation which is in the ms time scale. In such conditions, the crystallinity degree depends solely on the chemical nature of the polymer while the thickness of crystallites is correlated with the THF volume in the particles.

When the composition of the polymer/THF/water system is in the "unstable Ouzo" domain, most of the particles are unstable and sediment as shown by the low polymer recovery in the supernatants. The aggregates entrap a large amount of THF which is not favorable for subsequent crystallization of the polymer (Table 1). However, the few nanoparticles that remain stable under such conditions have probably the same crystalline properties to those formed in the Ouzo domain as evidenced by their values of melting points (Figure 6.A). Finally, the reversible crystallization of PCL upon removal of THF by dialysis emphasizes the equilibrium state of the polymer chains in particles. In other words, polymer crystals are 'living crystals' like in melting crystallization $^{52}$ despite the metastable state of the nanoparticles in the Ouzo domain.

\section{CONCLUSION}

The formation of polymer particles by solvent displacement method is a non-equilibrium process with a sensitive dependence of the particle properties on initial conditions such as mixing time. Here the nanoprecipitation of PCL from polymer solutions in THF was studied through fast and drop-by-drop addition (FA and DD) of water in the organic phase. The so-called Ouzo domain featuring the presence of metastable nanoparticles has been demonstrated for both mixing methods at high supersaturation and low polymer concentration. However, the FA method accounts for a wider Ouzo domain and the particles obtained inside are smaller and more stable against aging than those produced with the DD approach method whatever the THF fraction considered. This is consistent with a nucleation and growth (or aggregation) mechanism 
where the FA method allows for fast and homogeneous supersaturation conditions that favor the nucleation rate and thus the formation of small and uniform particles. For the DD method, the composition trajectories systematically cross the "unstable Ouzo" region before reaching the stable Ouzo domain. This leads to heterogeneous nucleation at low level of supersaturation favoring the formation of larger and more polydisperse particles. Regarding the crystalline properties of particles, they are not affected by the mixing process. The melting point of the polymer has the same linear dependence with the THF fraction in the medium for the FA and DD approaches. The crystallinity is also constant around $20 \%$ for both mixing methods and regardless of the molecular weight of the polymer. Therefore, this suggests that crystallization occurs after particle formation and depends solely on the chemical nature of the polymer. In the "unstable Ouzo" region, large amorphous aggregates highly swollen by THF coexist with a small population of nanoparticles featuring same crystalline properties as those formed in the Ouzo domain. Importantly, the semi-crystalline properties of bulk PCL can be recovered by removing THF molecules entrapped in the particles during a dialysis step. This underlines the equilibrium nature of the crystalline domains of the polymer unlike the non-equilibrium state of the particles formed by solvent displacement.

\section{ASSOCIATED CONTENT}

Supporting Information. Data available includes details on additional NMR, DLS, SEM and $\mu \mathrm{DSC}$ experiments. (PDF)

\section{AUTHOR INFORMATION}

Corresponding authors 
*Email: pucci@,crpp-bordeaux.cnrs.fr; chapel@,crpp-bordeaux.cnrs.fr; $\underline{\text { schatz@enscbp.fr }}$

\section{AKNOWLEDGMENTS}

This research was supported by a grant from the IDEX-Bordeaux and by Agence Nationale de la Recherche (PANORAMA project, ANR-13-BS08-0015). The financial support from the CPER CAMPUSB project funded by the French state and the Region Nouvelle Aquitaine is gratefully acknowledged. The Czech-French mobility program Barrande is also acknowledged for providing financial support. The Leon Brillouin Laboratory is thanked for providing beamtime on Paxy.

\section{REFERENCES}

(1) Chan, J.M.; Valencia, P. M.; Zhang, L.; Langer, R.; Farokhzad, O. C. Polymeric Nanoparticles for Drug Delivery. Methods Mol. Biol. 2010, 624, 163-175.

(2) Pu, K.; Chattopadhyay, N.; Rao, J. Recent Advances of Semiconducting Polymer Nanoparticles in In Vivo Molecular Imaging. J Control Release 2016, 240, 312-322.

(3) Canfarotta, F.; Whitcombe, M. J.; Pilersky, S. A. Polymeric Nanoparticles for Optical Sensing. Biotechnol. Adv. 2013, 31, 1585-1599.

(4) Busseron, E.; Ruff, Y.; Moulin, E.; Giuseppone, N. Supramolecular Self-Assemblies as Functional Nanomaterials. Nanoscale 2013, 5, 7098-7140.

(5) Wang, Y.; He, J.; Liu, C.; Chong, W.; Chen, H. Thermodynamics versus Kinetics in Nanosynthesis. Angew. Chem. Int. Ed. 2015, 54, 2022-2051. 
(6) Qi, L.; Fresnais, J. ; Berret, J.-F.; Castaing, J.-C.; Grillo, I.; Chapel, J.-P. Influence of the Formulation Process in Electrostatic Assembly of Nanoparticles and Macromolecules in Aqueous Solution: The Mixing Pathway. J. Phys. Chem. C. 2010, 114, 12870-12877.

(7) Vitorazi, L.; Ould-Moussa, N.; Sekar, S.; Fresnais, J.; Loh, W.; Chapel, J.-P.; Berret, J.-F. Evidence of a Two-Step Process and Pathway Dependency in the Thermodynamics of Poly(diallyldimethylammonium chloride)/Poly(sodium acrylate) Complexation. Soft Matter $2014,10,9496-9505$.

(8) Zhang, L.; Eisenberg, A. Thermodynamic vs Kinetic Aspects in the Formation and Morphological Transitions of Crew-Cut Aggregates Produced by Self-Assembly of Polystyrene-b-poly(acrylic acid) Block Copolymers in Dilute Solution. Macromolecules 1999, 32, 2239-2249.

(9) Hayward, R. C.; Pochan, D. J. Tailored Assemblies of Block Copolymers in Solution: It Is All about the Process. Macromolecules 2010, 43, 3577-3584.

(10) Nicolai, T.; Colombani, O.; Chassenieux, C. Dynamic Polymeric Micelles versus Frozen Nanoparticles Formed by Block Copolymers. Soft Matter. 2010, 6, 3111-3118.

(11)Qi, L.; Fresnais, J.-F.; Berret, J.-F.; Castaing, J.-C.; Grillo, I.; Chapel, J.-P. Influence of the Formulation Process in Electrostatic Assembly of Nanoparticles and Macromolecules in Aqueous Solution: The Interaction Pathway. J. Phys. Chem. C. 2010, 114, 16373-16381.

(12)Horn, D.; Rieger, J. Organic nanoparticles in the aqueous phase-theory, experiment, and use. Angew. Chem. Int. Ed. 2001, 40, 4330-4361. 
(13) Lince, F.; Marchisio, D. L.; Barresi, A. A. Strategies To Control the Particle Size

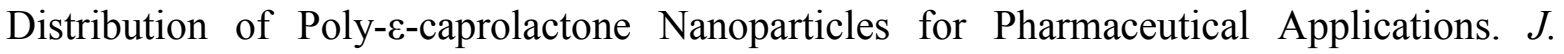
Colloid Interface Sci. 2008, 322, 505-515.

(14) Saada, W. S.; Prud'homme, R. K.; Principles of Nanoparticle Formation by Flash Nanoprecipitation. Nanotoday 2016, 11, 212-227.

(15) Matteucci, M. M.; Hotze, M. A.; Johnston, K. P.; Williams, R. O. III Drug Nanoparticles by Antisolvent Precipitation: Mixing Energy versus Surfactant Stabilization. Langmuir 2006, $22,8951-8959$.

(16) Aubry, J.; Ganachaud, F.; Cohen Addad, J.-P.; Cabane, B. Nanoprecipitation of Polymethylmethacrylate by Solvent Shifting: 1. Boundaries. Langmuir 2009, 25, 1970-1979.

(17) Mora-Huertas, C.E.; Fessi, H.; Elaissari, A. Influence of Process and Formulation Parameters on the Formation of Submicron Particles by Solvent Displacement and Emulsification-Diffusion Methods Critical Comparison. Adv. Colloid Interf. Sci. 2011, 163, $90-122$.

(18) Johnson, B. K.; Prud'homme, R.K. Mechanism for Rapid Self-Assembly of Block Copolymer Nanoparticles. Phys. Rev. Lett. 2003, 91, 118302- 118700.

(19) de Oliveira, A. M.; Jäger, E.; Jäger, A.; Stepanek, P.; Giacomelli, F.C. Physicochemical Aspects Behind the Size of Biodegradable Polymeric Nanoparticles: A Step Forward. Colloids Surf. A: Physicochem. Eng. Aspects 2013, 436, 1092-1102. 
(20)Bilati, U.; Allemann, E.; Doelker, E. Development of a Nanoprecipitation Method Intended for the Entrapment of Hydrophilic Drugs into Nanoparticles. Eur. J. Pharm. Sci. 2005, 24, $65-75$.

(21) Jeong, J.-C.; Lee, J.; Cho, W. Effects of Crystalline Microstructure on Drug Release Behavior of Poly( $\varepsilon$-caprolactone) Microspheres. J. Control. Release 2003, 92, 249-258.

(22)Hyvönen, S.; Peltonen, L.; Karjalainen, M.; Hirvonen, J. Effect of Nanoprecipitation on the Physicochemical Properties of Low Molecular Weight Poly(L-lactic acid) Nanoparticles Loaded with Salbutamol Sulphate and Beclomethasone Dipropionate. Int. J. Pharm. 2005, 295, 269-281.

(23)Cho, E. C.; Cho, K.; Ahn, J. K.; Kim, J. Chang, I.-S. Effect of Particle Size, Composition, and Thermal Treatment on the Crystalline Structure of Polycaprolactone Nanoparticles. Biomacromolecules 2006, 7, 1679-1685.

(24) Wu, C.; Jim, T. F.; Gan, Z.; Zhao, Z.; Wang, S. A Heterogeneous Catalytic Kinetics for Enzymatic Biodegradation of Poly( $\epsilon$-caprolactone) Nanoparticles in Aqueous Solution. Polymer 2000, 41, 3593-3597.

(25) Woodruff, M. A.; Hutmacher, D. W. The Return of a Forgotten Polymer: Polycaprolactone in the 21st Century. Prog. Polym. Sci. 2010, 35, 1217-1256.

(26) Schubert, S.; Delaney, J. T.; Schubert, U.S. Nanoprecipitation and Nanoformulation of Polymers: From History to Powerful Possibilities Beyond Poly(lactic acid). Soft Matter 2011, 7, 1581-1588. 
(27)Leroueil-Le Verger, M.; Fluckiger, L.; Kim, Y.-I.; Hoffman, M.; Maincent, P. Preparation and characterization of nanoparticles containing an antihypertensive agent. Eur. J. Pharm. Biopharm. 1998, 46, 137-143.

(28)Lannibois, H.; Hamsy, A.; Botet, R.; Aguerrre Chariol, O.; Cabane, B. Surfactant Limited Aggregation of Hydrophobic Molecules in Water. J. Phys. II France 1997, 7, 319-342.

(29) Lepeltier, E.; Bourgaux, C.; Couvreur, P. Nanoprecipitation and the "Ouzo Effect": Application to Drug Delivery Devices. Advanced Drug Delivery Reviews 2014, 71, 86-97.

(30) Stainmesse, S.; Orecchioni, A.-M.; Nakache, E.; Puisieux, F.; Fessi, H. Formation and stabilization of a biodegradable polymeric colloidal suspension of nanoparticles. Colloid Polym. Sci. 1995, 273, 505-511.

(31) Aminabhavi, T. M.; Gopalakrishna, B. Density, Viscosity, Refractive Index, and Speed of Sound in Aqueous Mixtures of N,N-Dimethylformamide, Dimethyl Sulfoxide, N,NDimethylacetamide, Acetonitrile, Ethylene Glycol, Diethylene Glycol, 1,4-Dioxane, Tetrahydrofuran, 2-Methoxyethanol, and 2-Ethoxyethanol at 298.15 K. J. Chem. Eng. Data. 1995, 40, 856-861.

(32) Koenig, M. F.; Huang S. J. Biodegradable blends and composites of polycaprolactone and starch derivatives. Polymer 1995, 36, 1877-1882.

(33)Crescenzi, V.; Manzini, G.; Calzolari, G.; Borri, C. Thermodynamics of fusion of poly- $\beta-$ propiolactone and poly-e-caprolactone. Comparative analysis of the melting of aliphatic polylactone and polyester chains. Eur. Polym. J. 1972, 8, 449-463. 
(34) Ganachaud, F.; Katz, J.L. Nanoparticles and Nanocapsules Created using the Ouzo Effect/ Spontaneous Emulsification as an Alternative to Ultrasonic and High-Shear Devices. ChemPhysChem 2005, 6, 209-216.

(35) Botet, R. The "Ouzo Effect", Recent Developments and Application to Therapeutic Drug Carrying. J. Phys. Conf. Ser. 2012, 352, 012047.

(36) Vitale, S.A.; Katz, J.L. Liquid Droplet Dispersions Formed by Homogeneous Liquid-Liquid Nucleation: "The Ouzo Effect”. Langmuir. 2003, 19, 4105-4110.

(37)Lebouille, J.G.J.L.; Stepanyan, R.; Slot, J.J.M., Cohen Stuart M.A., Tuinier, R. Nanoprecipitation of Polymers in a Bad Solvent. Colloids Surf. A Physicochem. Eng. Asp. 2014, 460, 225-235.

(38) Roger, K.; Eissa, M.; Elaissari, A.; Cabane, B. Surface Charge of Polymer Particles in Water: The Role of Ionic End-Groups. Langmuir 2013, 29, 11244-11250.

(39) Roger, K.; Cabane, B. Uncontaminated Hydrophobic/Water Interfaces Are Uncharged: A Reply. Angew. Chem. 2012, 51, 12943-12945.

(40) Roger, K.; Botet, R.; Cabane, B. Coalescence of Repelling Colloidal Droplets: A Route to Monodisperse Populations. Langmuir 2013, 29, 5689-5700.

(41)Brick, M. C.; Palmer, H. J.; Whitesides, T. H. Formation of Colloidal Dispersions of Organic Materials in Aqueous Media by Solvent Shifting. Langmuir 2003, 19, 6367-6380.

(42) Flory, P. J. Principles of polymer chemistry. Itaha, New York: Cornell University Press, 1953. 
(43) Belfiore, L. A. Physical Properties of Macromolecules. s.1.: John Wiley \& Sons, Inc, 2010.

(44) Labet, M.; Thielemans, W. Synthesis of polycaprolactone: a review. Chem. Soc. Rev. 2009, $38,3484-3504$.

(45) Yilgör, E.; Isik, M.; Söz, C. K.; Yilgör, I. Synthesis and structure-property behavior of polycaprolactone-polydimethylsiloxane-polycaprolactone triblock copolymers. Polymer 2016, $83,138-153$.

(46) Chen, E. C.; Wu, T. M. Isothermal crystallization kinetics and thermal behavior of poly(3caprolactone)/multi-walled carbon nanotube composites. Polym. Degrad. Stab. 2007, 92, 1009-1015.

(47)Gummel, J.; Boué, F.; Demé, B.; Cousin F. Charge Stoichiometry Inside Polyelectrolyte-Protein Complexes: A Direct SANS Measurement for the PSSNa-Lysozyme System. J. Phys. Chem. B. 2006, 110, 24837-24846.

(48) Richards, J. J.; Whittle, C. L.; Guozheng, S.; Pozzo, L. D. Correlating Structure and Photocurrent for Composite Semiconducting Nanoparticles with Contrast Variation SmallAngle Neutron Scattering and Photoconductive Atomic Force Microscopy. ACS Nano 2014, $8,4313-4324$.

(49) Huang, Y.; Xu, X.; Luo, X.; Ma, D. Molecular Weight Dependence Of The Melting Behavior Of Poly(ع-Caprolactone). Chin. J. Polym. Sci. 2002, 20, 45-51.

(50) Bordes, C.; Fréville, V.; Ruffin, E.; Marote, P.; Gauvrit, J. Y.; Briançon, S.; Lantéri, P. Determination of poly(E-caprolactone) solubility parameters: Application to solvent substitution in a microencapsulation process. Int. J. Pharm. 2010, 383, 236-243. 
(51)Jiang, Q.; Yang, C. C.; Li, J. C. Size-Dependent Melting Temperature of Polymers. Macromol. Theory Simul. 2003, 12, 57-60.

(52)Wurm, A.; Merzlyakov, M.; Schick, C. Reversible Melting During Crystallization of Polymers Studied by Temperature Modulated Techniques (TMDSC, TMDMA). J. Therm. Anal. Calorim. 2000, 60, 807-820. 

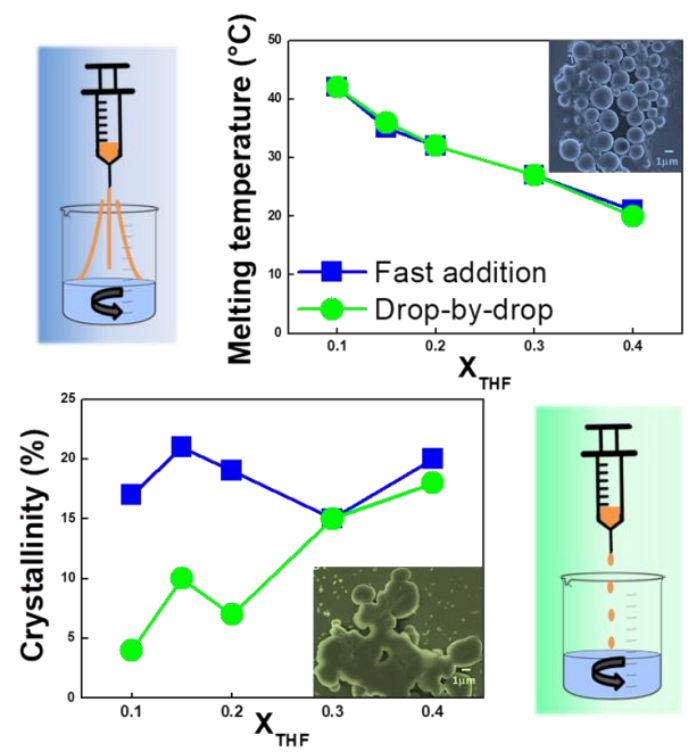